\title{
Design, synthesis and computational validation of novel benzimidazole/indole-based PPAR $\alpha$ and PPAR $\gamma$ partial agonists
}

\author{
RAMAN K VERMA ${ }^{\mathrm{a}, *}$, PRITHWISH GHOSH ${ }^{\mathrm{a}}$, VIJAY KUMAR ${ }^{\mathrm{a}}$ and LALIT K WADHWA ${ }^{\mathrm{b}}$ \\ a Synthetic Organic and Medicinal Chemistry Laboratory, Department of Chemistry, Punjabi University, \\ Patiala 147 002, India \\ bInd-Swift Laboratories Limited (Research and Development Centre), Plot No. E-5, Industrial Area, Phase-2, \\ SAS Nagar, Mohali 160 055, India \\ e-mail: raman_verma58@yahoo.com
}

MS received 28 June 2012; revised 15 March 2013; accepted 4 July 2013

\begin{abstract}
The design and synthesis of benzimidazolyl and indolyl linked $\alpha$-alkoxy phenylpropanoic acid derivatives and the $\beta$-keto ester analogues in an effort to develop novel peroxisome proliferator activated receptors ligands expected to exhibit PPAR $\alpha$ and $\operatorname{PPAR} \gamma$ partial agonism in the management of hyperglycemia and hyperlipidemia for the treatment of type 2 diabetes is reported. Computational validation of the designed molecules through activity prediction and docking studies showed expected results.
\end{abstract}

Keywords. $\quad \beta$-keto ester; $\alpha$-alkoxy acid; PPAR; partial agonism.

\section{Introduction}

Type 2 diabetes mellitus is a disease of complex pathogenesis and pleiotropic clinical manifestations. The greatest clinical challenge in this disease is the prevention of long term complications, many of which involve cardiovascular problems. The peroxisome proliferatoractivated receptor $\alpha$ and $\gamma$ isoforms are pharmaceutical targets for therapeutic intervention as they can potentially ameliorate not only the hyperglycemia of diabetes but also the dyslipidemia that is characteristic of this disorder. ${ }^{1}$ Activation of PPAR $\alpha$ reduces triglycerides and is involved in regulation of energy homeostasis. Activation of PPAR $\gamma$ causes insulin sensitization and enhances glucose metabolism. The development of multimodal drugs which can reduce hyperglycemia and concomitantly inhibit the progression of secondary cardiovascular complications offer valuable therapeutic option. ${ }^{2}$ PPAR $\alpha$ activators primarily improve dyslipidemia, whereas thiazolidinediones are potent PPAR $\gamma$ activators that improve insulin resistance though are not devoid of side effects. ${ }^{3}$

The PPAR $\alpha / \gamma$ dual agonists (figure 1) are developed to increase insulin sensitivity and simultaneously prevent diabetic cardiovascular complications. ${ }^{3}$ Among the dual activators, glitazars elicited high hopes and deep disappointment as potential new drugs. Although

*For correspondence they demonstrated beneficial impact over individual PPAR agonists by improving lipid and glucose homeostasis both, safety had been a critical issue and derailed their development because of adverse toxicity profiles. Muraglitazar and tesaglitazar (figure 1) though reached advanced phases of clinical trials but were noted to produce several cardiovascular risks and carcinogenicity. ${ }^{4,5}$

Disappointingly, full PPAR agonists have been plagued by certain adverse side effects. On the up side, partial PPAR agonists have the potential to retain the desired efficacy and beneficial effects of full PPAR agonists while diminishing the unwanted effects. ${ }^{6}$ In vitro studies by researchers from Astellas and Roche also indicated that their PPAR $\gamma$ partial agonists activate pathways that ameliorate insulin resistance without stimulating fat accumulation in adipocytes. ${ }^{7,8}$

Partial agonism to both PPAR $\alpha$ and PPAR $\gamma$ receptors by dual activators may provide a solution resulting in the desirable responses and reducing the adverse effects caused by the individual agonists for the treatment of T2DM. 3D QSAR methods combined with docking studies provide valuable insights to determine the significant structural features required for optimum activity in designing of new candidates. The subsequent prediction and comparative analysis of activities and binding affinities of the designed molecules help to select most suitable candidates for further studies. The work reported here presents such an approach. The design (maintaining the structural requirements of PPAR ligands), prediction of PPAR $\alpha$ and PPAR $\gamma$ 

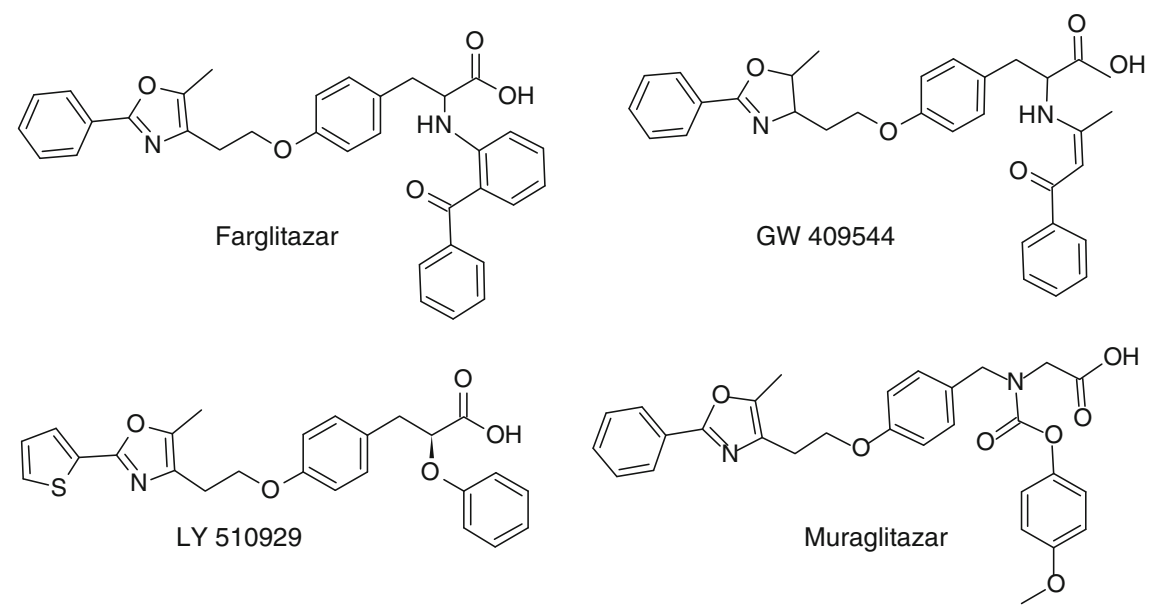<smiles>CCOC(Cc1ccc(OCCn2c3ccccc3c3ccccc32)cc1)C(=O)O</smiles><smiles>CCOC(Cc1ccc(OCCc2ccc(OS(C)(=O)=O)cc2)cc1)C(=O)O</smiles><smiles>CCOC(Cc1ccc(OCCN2c3ccccc3Oc3ccccc32)cc1)C(=O)O</smiles><smiles>CC(=O)c1ccccc1NC(Cc1ccc(OCCc2nc(-c3ccccc3)oc2C)cc1)C(=O)O</smiles>

Figure 1. Molecular structure of some well-studied PPAR dual agonists.

activities, prediction of binding affinities of a set of novel molecules as PPAR $\alpha / \gamma$ partial agonists and their syntheses have been reported in the present work.

\section{Experimental}

\subsection{Materials and methods}

All the chemicals used were purchased from Aldrich and Sdfine chemicals. The melting/boiling points reported here were recorded using an open conc. sulphuric acid bath and are uncorrected. The infrared and ${ }^{1} \mathrm{H}$ NMR spectra of the reported compounds were recorded on Perkin-Elmer Spectrum RX FTIR Spectrophotometer, and AC400F, $400 \mathrm{MHz}$ Bruker spectrometer, respectively at RSIC, Panjab University, Chandigarh. LCMS of the compounds were recorded on LCMS LCQ Finnigan Matt (APCI + ve mode) at Central Instrumentation Lab, NIPER, SAS Nagar, Mohali, Punjab. GCMS and elemental analysis of these compounds were carried out on Shimadzu GCMS-QP2010 Plus and Vario Micro CHN Elemental Analyzer, respectively at Instrumental Laboratory, Department of Chemistry, Punjabi University, Patiala.

\subsection{Synthetic procedures for the lhs units}

2.2a (1-Methyl-1H-benzimidazol-2-yl)methanol (compound 1, scheme 1): N-methyl-1,2-phenylenediamine dihydrochloride $(11.700 \mathrm{~g}, 60 \mathrm{mmol})$, glycolic acid (17 mL, $180 \mathrm{mmol}, 65 \%$ aqueous solution) and water<smiles>CNc1ccccc1N</smiles>

Scheme 1. Reagents and conditions: (a) glycolic acid, water, reflux; (b) 4-fluorobenzaldehyde, $\mathrm{NaH}, \mathrm{DMF}, 0-25^{\circ} \mathrm{C}$. 
(42 $\mathrm{mL})$ were combined, and refluxed for $90 \mathrm{~min}$. The reaction mixture was basified with dilute ammonia solution under ice cold condition after cooling to room temperature. The grey solid separated was collected under suction, washed with cold water, and which upon recrystallization from methanol gave $(8.75 \mathrm{~g}, 90 \%)$ of 1a: mp $145-147^{\circ} \mathrm{C}$; FTIR (KBr): 3660, 3450-3200, 3142, 2950, 2842, 1 548, 1482, 1434, 1357, $739 \mathrm{~cm}^{-1}$; ${ }^{1} \mathrm{H}$ NMR $\left(\mathrm{CDCl}_{3}+\mathrm{DMSO}_{-} \mathrm{d}_{6}\right) \delta: 7.69(\mathrm{~m}, 1 \mathrm{H}), 7.27$ (m, 3H), 4.89 (s, 2H), 3.82 (s, 3H).

2.2b Ethyl 1-methyl-1H-indole-2-carboxylate (compound 2, scheme 2): To a stirred solution of sodium hydride $(1.100 \mathrm{~g}, 27.5 \mathrm{mmol}, 60 \% \mathrm{w} / \mathrm{w})$ in dry DMF $(100 \mathrm{ml})$ was added ethyl indole-2-carboxylate $(4.160 \mathrm{~g}, 22 \mathrm{mmol})$ at $2-5^{\circ} \mathrm{C}$, and the mixture was stirred for $30 \mathrm{~min}$ at room temperature (rt) $\left(\mathrm{ca} .30^{\circ} \mathrm{C}\right)$. A solution of iodomethane $(3.750 \mathrm{~g}, 26.4 \mathrm{mmol})$ in dry DMF $(5 \mathrm{~mL})$ was added drop-wise over $15 \mathrm{~min}$ at $2-$ $5^{\circ} \mathrm{C}$, and stirred for $28 \mathrm{~h}$ at $\mathrm{rt}$. The reaction mixture was quenched with ice-water, and extracted with EtOAc $(3 \times 25 \mathrm{~mL})$. The combined organic extracts were washed with brine and water, dried, and concentrated. The crude product was treated with hexane to obtain the pure product 2 as a white solid $(3.35 \mathrm{~g}, 75 \%)$ : $\mathrm{mp}$ 68-70 ${ }^{\circ} \mathrm{C}$; FTIR (KBr): 2982, 2850, 1703, 1515, 1469 , 1400, 1378, 1251, 1087, $747 \mathrm{~cm}^{-1} ;{ }^{1} \mathrm{H}$ NMR $\left(\mathrm{CDCl}_{3}\right)$ $\delta: 7.67(\mathrm{~d}, J=7.9 \mathrm{~Hz}, 1 \mathrm{H}), 7.40(\mathrm{~m}, 1 \mathrm{H}), 7.33(\mathrm{~m}, 1 \mathrm{H})$, $7.30(\mathrm{~s}, 1 \mathrm{H}), 7.14(\mathrm{~m}, 1 \mathrm{H}), 4.37(\mathrm{q}, J=7.1 \mathrm{~Hz}, 2 \mathrm{H})$, $4.08(\mathrm{~s}, 3 \mathrm{H}), 1.41(\mathrm{t}, J=7.2 \mathrm{~Hz}, 3 \mathrm{H})$.

2.2c (1-Methyl-1H-indol-2-yl)methanol (compound $\mathbf{2 a}$, scheme 2): To a stirred suspension of lithium aluminium hydride $(7.600 \mathrm{~g}, 200 \mathrm{mmol})$ in THF $(100 \mathrm{~mL})$ was added $2(4.060 \mathrm{~g}, 20 \mathrm{mmol})$ in THF $(5 \mathrm{~mL})$, and the mixture was stirred at room temperature (ca. $30^{\circ} \mathrm{C}$ ) for $36 \mathrm{~h}$. The reaction mixture was quenched with ice water, acidified with $2 \mathrm{~N}-\mathrm{HCl}$, and extracted with ethyl acetate $(3 \times 20 \mathrm{ml})$. The combined organic extracts were dried over sodium sulphate and concentrated to give white solid and which upon recrystallization from ethyl acetate gave $(3.06 \mathrm{~g}, 95 \%)$ of $\mathbf{2 a}$ : mp 108-110 ${ }^{\circ}$; FTIR (KBr): 3600-3400, 2962, 2929 , $2850,1612,1515,1468,1399,745 ;{ }^{1} \mathrm{H}$ NMR $\left(\mathrm{CDCl}_{3}\right)$ $\delta: 7.58(\mathrm{~d}, J=7.9 \mathrm{~Hz}, 1 \mathrm{H}), 7.32(\mathrm{~m}, 1 \mathrm{H}), 7.23(\mathrm{~m}, 1 \mathrm{H})$, $7.10(\mathrm{~m}, 1 \mathrm{H}), 6.45(\mathrm{~s}, 1 \mathrm{H}), 4.80(\mathrm{~s}, 2 \mathrm{H}), 3.80(\mathrm{~s}, 3 \mathrm{H})$.

\subsection{General procedure for the syntheses of heterocyclyl linked benzaldehydes (compounds $\mathbf{1 a}$ and $\mathbf{2 b}$ )}

To a stirred suspension of sodium hydride $(1.4 \mathrm{mmol}$, $60 \% \mathrm{w} / \mathrm{w}$ dispersion $)$ in dry DMF $(20 \mathrm{~mL})$ was added 1/2a $(1.2 \mathrm{mmol})$ in dry DMF $(5 \mathrm{~mL})$ at $0^{\circ} \mathrm{C}$, and the mixture was stirred for $30 \mathrm{~min}$ at $\mathrm{rt}\left(\mathrm{ca} .30^{\circ} \mathrm{C}\right)$. A solution of 4-fluorobenzaldehyde $(1.3 \mathrm{mmol})$ in dry DMF $(5 \mathrm{~mL})$ was added drop-wise over $15 \mathrm{~min}$ at $0^{\circ} \mathrm{C}$, and stirred for $24 \mathrm{~h}$ at rt. The reaction mixture was quenched with water and extracted with EtOAc $(3 \times 30 \mathrm{~mL})$. The combined organic extracts were washed with brine, dried over anhydrous $\mathrm{Na}_{2} \mathrm{SO}_{4}$, and concentrated. The residue was chromatographed to achieve the target benzaldehyde.

2.3a 4-[(1-Methyl-1H-benzimidazol-2-yl)methoxy]benzaldehyde (compound 1a, scheme 1): Yield: $30 \%$. Mp: $120-122^{\circ} \mathrm{C}$; FTIR (KBr): 2926, 2823, 2733, 1697, 1601, 1577, 1482, 1430, 1363, 1247,1005, $828 \mathrm{~cm}^{-1}$; ${ }^{1} \mathrm{H} \mathrm{NMR}\left(\mathrm{CDCl}_{3}\right) \delta: 9.9(\mathrm{~s}, 1 \mathrm{H}), 7.83(\mathrm{~m}, 3 \mathrm{H}), 7.39$ $(\mathrm{m}, 3 \mathrm{H}), 7.24(\mathrm{~d}, J=8.8 \mathrm{~Hz}, 2 \mathrm{H}), 5.59(\mathrm{~s}, 2 \mathrm{H}), 3.94$ (s, 3H) ; GCMS (m/z): $266[\mathrm{M}]^{+}$, 145; Anal. Calcd for $\mathrm{C}_{16} \mathrm{H}_{14} \mathrm{~N}_{2} \mathrm{O}_{2}$ : C (72.16\%), $\mathrm{H}(5.30 \%), \mathrm{N}(10.52 \%)$; Found: C (72.51\%), H (5.57\%), N (10.19\%).

$2.3 \mathrm{~b}$ 4-[(1-Methyl-1H-indol-2-yl)methoxy]benzaldehyde (compound $\mathbf{2 b}$, scheme 2): Yield: 30\%. Mp: 158-160 ${ }^{\circ}$; FTIR (KBr): 2933, 2823, 2733, 1697, 1598, 1506, 1468, 1380, 1244, 1160, $828 \mathrm{~cm}^{-1} ;{ }^{1} \mathrm{H}$ NMR $\left(\mathrm{CDCl}_{3}\right) \delta: 9.90(\mathrm{~s}, 1 \mathrm{H}), 7.86(\mathrm{~d}, J=8.7 \mathrm{~Hz}$, $2 \mathrm{H}), 7.62(\mathrm{~d}, J=7.8 \mathrm{~Hz}, 1 \mathrm{H}), 7.34(\mathrm{~m}, 1 \mathrm{H}), 7.27$ $(\mathrm{m}, 2 \mathrm{H}), 7.13(\mathrm{~d}, J=8.8 \mathrm{~Hz}, 2 \mathrm{H}), 6.63(\mathrm{~s}, 1 \mathrm{H}), 5.29$ (s, 2H), $3.82(\mathrm{~s}, 3 \mathrm{H})$; LCMS (m/z): $266[\mathrm{M}+1]^{+}$; Anal. Calcd for $\mathrm{C}_{17} \mathrm{H}_{15} \mathrm{NO}_{2}$ : C (76.96\%), H (5.70\%), N (5.28\%); Found: C (76.58\%), H (5.55\%), N (5.60\%).

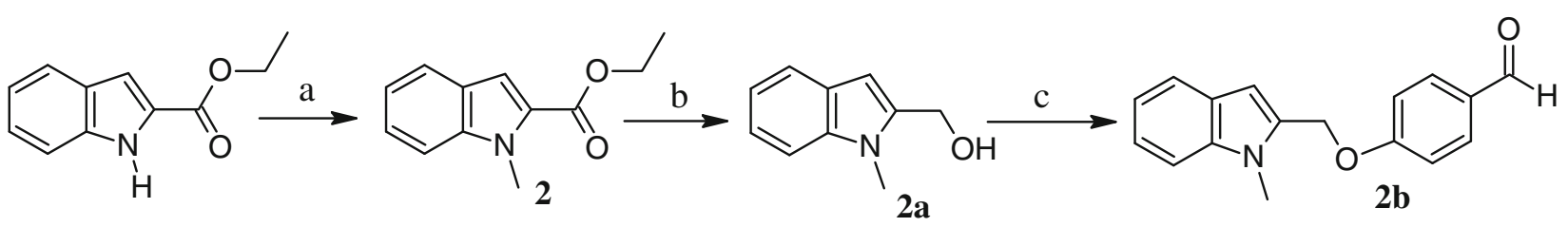

Scheme 2. Reagents and conditions: (a) NaH, DMF, MeI, rt; (b) LAH, THF, rt; (c) 4-fluorobenzaldehyde, NaH, DMF, $0-25^{\circ} \mathrm{C}$. 


\subsection{Synthetic procedures for the rhs units}

2.4a General procedure for the syntheses of $\mathbf{3 a}$ and $3 \boldsymbol{b}$ : A solution of $p$-anisaldehyde $(8.16 \mathrm{~g}, 60 \mathrm{mmol})$ and methyl acetoacetate/ethyl acetoactate $(60 \mathrm{mmol})$ in toluene $(150 \mathrm{~mL})$ containing a catalytic quantity of piperidinium acetate $(2 \mathrm{~mL})$ was refluxed in a DeanStark trap for $16 \mathrm{~h}$. After cooling to room temperature, the solution was concentrated. The residue was purified by column chromatography using a mixture of EtOAc and hexane (1:3) to give $\mathbf{3 a} \mathbf{a} / \mathbf{b}$ as brown viscous mass.

2.4b Methyl 2-(4-methoxy benzylidene)-3-oxobutanoate (compound 3a, scheme 3): Yield: 40.0\%. FTIR $\left(\mathrm{CHCl}_{3}\right): 2981,2841,1734,1640,1027,845 \mathrm{~cm}^{-1} ;{ }^{1} \mathrm{H}$ NMR $\left(\mathrm{CDCl}_{3}\right)$ mixture of geometric isomers, $\delta: 7.45$ (superimposed s, $1 \mathrm{H}+1 \mathrm{H}), 7.33(\mathrm{~d}, J=8.7 \mathrm{~Hz}, 2 \mathrm{H})$, $7.28(\mathrm{~d}, J=8.9 \mathrm{~Hz}, 2 \mathrm{H}), 6.84(\mathrm{~d}, J=8.8 \mathrm{~Hz}, 2 \mathrm{H}), 2.31$ (s, 3H), $6.82(\mathrm{~d}, J=9.3 \mathrm{~Hz}, 2 \mathrm{H}), 3.79$ (superimposed s, $3 \mathrm{H}+3 \mathrm{H}), 3.75(\mathrm{~s}, 3 \mathrm{H}), 3.74(\mathrm{~s}, 3 \mathrm{H}), 2.32(\mathrm{~s}, 3 \mathrm{H})$; GCMS (m/z): $234[\mathrm{M}]^{+}, 219,203$; Anal. Calcd for $\mathrm{C}_{13} \mathrm{H}_{14} \mathrm{O}_{4}$ : C(66.66\%), H(6.02\%); Found: C(66.51\%). H(6.20\%).

2.4c Ethyl 2-(4-methoxybenzylidene)-3-oxobutanoate (compound 3b, scheme 3): Yield: $38 \%$. FTIR $\left(\mathrm{CHCl}_{3}\right)$ : 2981, 2841, 1726, 1659, 1120, 1058, $830 \mathrm{~cm}^{-1}$; ${ }^{1} \mathrm{HNMR}\left(\mathrm{CDCl}_{3}\right)$ mixture of geometric isomers, $\delta: 7.50$ (s, 1H), $7.41(\mathrm{~s}, 1 \mathrm{H}), 7.33(\mathrm{~d}, J=8.8 \mathrm{~Hz}, 2 \mathrm{H}), 7.26(\mathrm{~d}$, $J=8.8 \mathrm{~Hz}, 2 \mathrm{H}$ ), 6.80 (overlapped d, $J=8.8 \mathrm{~Hz}, 2 \mathrm{H}$ ), $4.27(\mathrm{q}, J=7.1 \mathrm{~Hz}, 2 \mathrm{H}), 4.18(\mathrm{q}, J=7.1 \mathrm{~Hz}, 2 \mathrm{H}), 3.73$ (s, 3H), 3.72 (s, 3H), 2.30 (s, 3H), 2.29 (s, 3H), 1.22 (overlapped t, $J=7.1 \mathrm{~Hz}, 3 \mathrm{H}+3 \mathrm{H})$; $\operatorname{GCMS}(\mathrm{m} / \mathrm{z})$ : $248[\mathrm{M}]^{+}$, 233; Anal. Calcd for $\mathrm{C}_{14} \mathrm{H}_{16} \mathrm{O}_{4}: \mathrm{C}(67.73 \%)$, $\mathrm{H}(6.50 \%)$; Found: $\mathrm{C}(68.11 \%), \mathrm{H}(6.78 \%)$.

2.4d General procedure for the syntheses of $4 \boldsymbol{a}$ and $\mathbf{4 b}$ : To a solution of $\mathbf{3 a} / \mathbf{b}(12.8 \mathrm{mmol})$ in DCM at $0^{\circ} \mathrm{C}$ was added $\mathrm{BBr}_{3}$ in $\mathrm{DCM}(13 \mathrm{~mL}, 1 \mathrm{M}$ solution) drop-wise while stirring and stirring continued for two $\mathrm{h}$ at $0^{\circ} \mathrm{C}$ and the at $\mathrm{rt}$ for $16 \mathrm{~h}$. The contents treated with ice cold water and the organic layers separated, washed with brine, dried over sodium sulphate, filtered and solvent evaporated and the residue purified by column chromatography EtOAc/Hexane (1:4) to yield the target demethylated $\beta$-ketoester (4a/b) as yellow solid.

2.4e Methyl 2-(4-hydroxybenzylidene)-3-oxobutanoate (compound 4a, scheme 3): Yield: 37\%. Mp: 152155 ${ }^{\circ} \mathrm{C}$; FTIR (KBr): 3349, 2987, 2940, 1731, 1645, 1572, 1168, 1026, $856 \mathrm{~cm}^{-1} ;{ }^{1} \mathrm{H}$ NMR $\left(\mathrm{CDCl}_{3}\right) \mathrm{mix}-$ ture of $E$ and $Z$ isomers, $\delta: 7.55(\mathrm{~s}, 1 \mathrm{H}), 7.44(\mathrm{~s}, 1 \mathrm{H})$, $7.26(\mathrm{~d}, J=8.7 \mathrm{~Hz}, 2 \mathrm{H}), 7.23(\mathrm{~d}, J=8.7 \mathrm{~Hz}, 2 \mathrm{H}), 6.75$ $(\mathrm{d}, J=8.7 \mathrm{~Hz}, 2 \mathrm{H}), 6.74(\mathrm{~d}, J=8.7 \mathrm{~Hz}, 2 \mathrm{H}), 5.47$ (s, 1H), $5.27(\mathrm{~s}, 1 \mathrm{H}), 3.80$ (s, 3H), 3.75 (s, 3H), 2.33 (s, 3H), 2.31 (s, 3H); GCMS (m/z): $220[\mathrm{M}]^{+}, 205,189$, 160; Anal. Calcd for $\mathrm{C}_{12} \mathrm{H}_{12} \mathrm{O}_{4}: \mathrm{C}(65.45 \%), \mathrm{H}(5.49 \%)$; Found: $\mathrm{C}(65.27 \%), \mathrm{H}(5.33 \%)$.

2.4f Ethyl 2-(4-hydroxybenzylidene)-3-oxobutanoate (compound 4b, scheme 3): Yield: 35\%. Mp: 134$136^{\circ} \mathrm{C}$; FTIR (KBr): 3360, 2982, 1715, 1665, 1114,

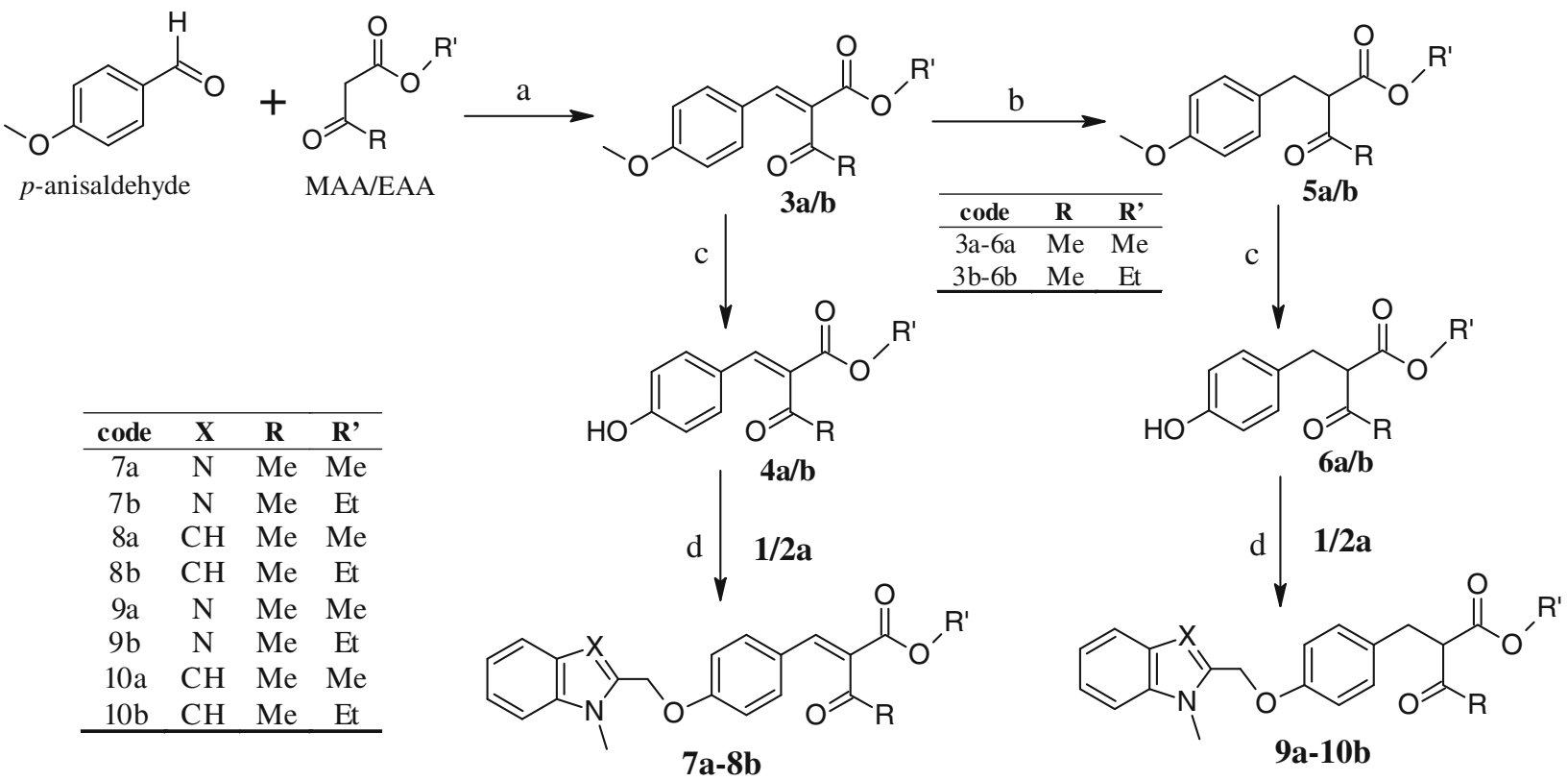

Scheme 3. Reagents and conditions: (a) Piperidinium acetate, toluene, reflux; (b) $\mathrm{Pd} / \mathrm{C}, \mathrm{H}_{2}, 20 \mathrm{psi}$, rt; (c) $\mathrm{BBr}_{3}$ in DCM, 0-25 $5^{\circ}$; (d) DEAD, $\mathrm{Ph}_{3} \mathrm{P}, \mathrm{THF}$, rt. 
1043, $834 \mathrm{~cm}^{-1}$; ${ }^{1} \mathrm{HNMR}\left(\mathrm{CDCl}_{3}\right)$ mixture of geometric isomers $(1: 3), \delta: 7.59(\mathrm{~s}, 1 \mathrm{H}), 7.49(\mathrm{~s}, 1 \mathrm{H}), 7.37$ $(\mathrm{d}, J=8.6 \mathrm{~Hz}, 2 \mathrm{H}+2 \mathrm{H}), 7.29(\mathrm{~d}, J=8.6 \mathrm{~Hz}, 2 \mathrm{H})$, $6.83(\mathrm{~d}, J=8.7 \mathrm{~Hz}, 2 \mathrm{H}), 6.80(\mathrm{~d}, J=8.7 \mathrm{~Hz}, 2 \mathrm{H}$, Ar), $4.36(\mathrm{q}, J=7.1 \mathrm{~Hz}, 2 \mathrm{H}), 4.29(\mathrm{q}, J=7.2 \mathrm{~Hz}$, $2 \mathrm{H}), 2.40(\mathrm{~s}, 3 \mathrm{H}), 2.39(\mathrm{~s}, 3 \mathrm{H}), 1.32$ (overlapped t, $J=$ $7.1 \mathrm{~Hz}, 3 \mathrm{H}+3 \mathrm{H})$; GCMS (m/z): $234[\mathrm{M}]^{+}, 219$; Anal. Calcd for $\mathrm{C}_{13} \mathrm{H}_{14} \mathrm{O}_{4}$ : $\mathrm{C}(66.66 \%), \mathrm{H}(6.02 \%)$; Found: $\mathrm{C}(66.14 \%), \mathrm{H}(6.58 \%)$.

$2.4 \mathrm{~g}$ General procedure for the syntheses of $5 \mathrm{a}$ and $5 \boldsymbol{b}$ : A solution of $\mathbf{3 a} / \mathbf{b}(12 \mathrm{mmol})$ in methanol $(80 \mathrm{~mL})$ in the presence of $10 \%$ palladium on charcoal $(0.5 \mathrm{~g})$ was shaken under an atmosphere of hydrogen (20 psi) in a Parr catalytic hydrogenator at room temperature until hydrogen uptake ceased $(>20 \mathrm{~h})$. The solution was filtered through celite, and the filtrate was evaporated under a vacuum. The residue was chromatographed eluting with a mixture of ethyl acetate and hexane (1:4) to give $\mathbf{4 a} \mathbf{a} \mathbf{b}$ as pale yellow viscous mass.

2.4h Methyl 2-(4-methoxybenzyl)-3-oxobutanoate (compound 5a, scheme 3): Yield: $67.8 \%$. FTIR $\left(\mathrm{CHCl}_{3}\right)$ : 2981, 2837, 1734, 1650, 1033, $833 \mathrm{~cm}^{-1} ;{ }^{1} \mathrm{H}$ NMR $\left(\mathrm{CDCl}_{3}\right) \delta: 7.08(\mathrm{~d}, J=8.7 \mathrm{~Hz}, 2 \mathrm{H}), 6.81(\mathrm{~d}, J=$ $8.7 \mathrm{~Hz}, 2 \mathrm{H}), 3.75(\mathrm{t}, J=6.9 \mathrm{~Hz}, 1 \mathrm{H}), 3.71(\mathrm{~s}, 3 \mathrm{H}), 3.69$ (s, 3H), 3.10 (d, $J=7.6 \mathrm{~Hz}, 2 \mathrm{H}), 2.17$ (s, 3H); GCMS $(\mathrm{m} / \mathrm{z})$ : $236\left(\mathrm{M}^{+}\right), 205,193$, 177; Anal. Calcd for $\mathrm{C}_{13} \mathrm{H}_{16} \mathrm{O}_{4}$ : $\mathrm{C}(66.09 \%), \mathrm{H}(6.83 \%)$; Found: $\mathrm{C}(66.17 \%)$, $\mathrm{H}(6.97 \%)$.

2.4i Ethyl 2-(4-methoxybenzyl)-3-oxobutanoate (compound $5 \boldsymbol{b}$, scheme 3$)$ : Yield: $70 \%$. FTIR $\left(\mathrm{CHCl}_{3}\right)$ : 2981, 2837, 1739, 1716, 1248, 1110, 1063, $823 \mathrm{~cm}^{-1}$; ${ }^{1} \mathrm{HNMR}\left(\mathrm{CDCl}_{3}\right) \delta: 7.09(\mathrm{~d}, J=8.6 \mathrm{~Hz}, 2 \mathrm{H}), 6.80$ $(\mathrm{d}, J=8.6 \mathrm{~Hz}, 2 \mathrm{H}), 4.14(\mathrm{q}, J=7.1 \mathrm{~Hz}, 2 \mathrm{H}), 3.77$ $(\mathrm{s}, 3 \mathrm{H}), 3.73(\mathrm{t}, J=7.6 \mathrm{~Hz}, 1 \mathrm{H}), 3.09(\mathrm{~d}, J=7.7 \mathrm{~Hz}$, 2H), $2.18(\mathrm{~s}, 3 \mathrm{H}), 1.21(\mathrm{t}, J=8.00 \mathrm{~Hz}, 3 \mathrm{H})$; GCMS $(\mathrm{m} / \mathrm{z}): 250[\mathrm{M}]^{+}, 207$; Anal. Calcd for $\mathrm{C}_{14} \mathrm{H}_{18} \mathrm{O}_{4}$ : C(67.18\%), H(7.25\%); Found: C(66.74\%), H(6.92\%).

$2.4 \mathrm{j}$ General procedure for the syntheses of $\boldsymbol{6} \boldsymbol{a}$ and $\boldsymbol{6} \boldsymbol{b}$ : To a solution of $\mathbf{5 a} / \mathbf{b}(12.8 \mathrm{mmol})$ in DCM at $0^{\circ} \mathrm{C}$ was added $\mathrm{BBr}_{3}$ in DCM $(13 \mathrm{~mL}, 1 \mathrm{M}$ solution) drop-wise while stirring and stirring continued for two $\mathrm{h}$ at $0^{\circ} \mathrm{C}$ and the at $\mathrm{rt}$ for $16 \mathrm{~h}$. The contents treated with ice cold water and the organic layers separated, washed with brine, dried over sodium sulphate, filtered and solvent evaporated and the residue purified by column chromatography EtOAc/Hexane (1:4) to yield the target demethylated $\beta$-ketoester $(\mathbf{6 a} / \mathbf{b})$ as colourless viscous mass. 2.4k Methyl 2-(4-hydroxybenzyl)-3-oxobutanoate (compound $\mathbf{6 a}$, scheme 3$)$ : Yield: $35 \%$. FTIR $\left(\mathrm{CHCl}_{3}\right)$ : 3348, 2980, 2837, 1741, 1714, 1034, $849 \mathrm{~cm}^{-1} ;{ }^{1} \mathrm{H}$ $\operatorname{NMR}\left(\mathrm{CDCl}_{3}\right) \delta: 7.02(\mathrm{~d}, J=8.5 \mathrm{~Hz}, 2 \mathrm{H}), 6.73(\mathrm{~d}, J=$ $8.5 \mathrm{~Hz}, 2 \mathrm{H}), 5.49(\mathrm{~s}, 1 \mathrm{H}), 3.75(\mathrm{t}, J=7.8 \mathrm{~Hz}, 1 \mathrm{H}), 3.70$ (s, 3H), 3.09 (d, $J=7.7 \mathrm{~Hz}, 2 \mathrm{H}), 2.17$ (s, 3H); GCMS $(\mathrm{m} / \mathrm{z}): 223[\mathrm{M}+1]^{+}, 207,190,147$; Anal. Calcd for $\mathrm{C}_{12} \mathrm{H}_{14} \mathrm{O}_{4}$ : $\mathrm{C}(64.85 \%), \mathrm{H}(6.35 \%)$; Found: $\mathrm{C}(64.66 \%)$, $\mathrm{H}(6.52 \%)$.

2.41 Ethyl 2-(4-hydroxybenzyl)-3-oxobutanoate (compound $\mathbf{6 b}$, scheme 3): Yield: 35\%. FTIR $\left(\mathrm{CHCl}_{3}\right)$ : 3437, 2931, 1732, 1710, 1219, 1614, 1516, 1446, 1153, 1056, $826 \mathrm{~cm}^{-1} ;{ }^{1} \mathrm{HNMR}\left(\mathrm{CDCl}_{3}\right), \delta: 7.02(\mathrm{~d}, J=$ $8.5 \mathrm{~Hz}, 2 \mathrm{H}), 6.72(\mathrm{~d}, J=8.5 \mathrm{~Hz}, 2 \mathrm{H}), 4.12(\mathrm{q}, J=$ $7.2 \mathrm{~Hz}, 2 \mathrm{H}), 3.74(\mathrm{t}, J=7.7 \mathrm{~Hz}, 1 \mathrm{H}), 2.18(\mathrm{~s}, 3 \mathrm{H}), 3.08$ $(\mathrm{d}, J=7.7 \mathrm{~Hz}, 2 \mathrm{H}), 1.21(\mathrm{t}, J=7.1 \mathrm{~Hz}, 3 \mathrm{H})$; GCMS (m/z): $236[\mathrm{M}]^{+}, 193$, 163; Anal. Calcd for $\mathrm{C}_{13} \mathrm{H}_{16} \mathrm{O}_{4}$ : C(66.09\%), H(6.83\%); Found: C(66.44\%), H(6.65\%).

\subsection{General procedure for the syntheses of final $\beta$-ketoester based NCEs (compounds $7 \boldsymbol{a}-10 \boldsymbol{b}$ )}

A solution of diethyl azodicarboxylate $(0.9 \mathrm{mmol})$ and triphenylphosphine $(0.9 \mathrm{mmol})$ in THF $(5 \mathrm{~mL})$ at $0^{\circ} \mathrm{C}$ was added dropwise to a stirred mixture of $\mathbf{1 / 2 a}$ $(0.6 \mathrm{mmol})$ and $\mathbf{4 a} / \mathbf{b}$ or $\mathbf{6 a} / \mathbf{b}(0.72 \mathrm{mmol})$ in $\mathrm{THF}$ $(20 \mathrm{~mL})$ at $0^{\circ} \mathrm{C}$. The reaction was stirred at $0^{\circ} \mathrm{C}$ for an hour and then at $\mathrm{rt}$ for $16 \mathrm{~h}$ and the solvent removed in vacuo to dryness. The residue was taken up in ethyl acetate/ water (2:1). The organic layer was separated, dried over sodium sulphate, filtered and the solvent evaporated under reduced pressure. The residue was chromatographed using a mixture of either methanol and dichloromethane (1:99) (for $\mathbf{7 a} / \mathbf{b}$ and $\mathbf{9 a} / \mathbf{b}$ ) or EtOAC and hexane (1:4) (for $\mathbf{8 a} / \mathbf{b}$ and $\mathbf{1 0 a} / \mathbf{b}$ ) to give of the coupled product $(7 \mathbf{a} / \mathbf{b}$ or $8 \mathbf{a} / \mathbf{b}$ or $9 \mathbf{a} / \mathbf{b}$ or $10 a / b)$.

2.5a Methyl 2-\{4-[(1-methyl-1H-benzimidazol-2-yl) methoxy]benzylidene)-3-oxobutanoate (compound 7a, scheme 3): Yield: $22.6 \%$. Mp: $161-163^{\circ} \mathrm{C}$; FTIR (KBr): 2917 and 2850, 1735, 1630, 1092, $844 \mathrm{~cm}^{-1} ;{ }^{1} \mathrm{H}$ NMR $\left(\mathrm{CDCl}_{3}\right)$ mixture of $E$ and $Z$ isomers, $\delta: 7.78$ (m, $1 \mathrm{H}), 7.71(\mathrm{~m}, 1 \mathrm{H}), 7.53(\mathrm{~s}, 1 \mathrm{H}), 7.42(\mathrm{~s}, 1 \mathrm{H}), 7.32(\mathrm{~d}$, $J=8.9 \mathrm{~Hz}, 2 \mathrm{H}+2 \mathrm{H}), 7.27(\mathrm{~m}, 3 \mathrm{H}+3 \mathrm{H}), 7.04(\mathrm{~d}, J=$ $8.9 \mathrm{~Hz}, 2 \mathrm{H}), 7.01(\mathrm{~d}, J=8.9 \mathrm{~Hz}, 2 \mathrm{H}), 5.36(\mathrm{~s}, 2 \mathrm{H}), 5.35$ (s, 2H), 3.78 (s, 3H), $3.75(\mathrm{~s}, 3 \mathrm{H}), 3.82(\mathrm{~s}, 3 \mathrm{H}), 3.81$ (s, 3H), 2.32 (s, 3H), 2.28 (s, 3H); LCMS (m/z): 366 $\left[\mathrm{M}^{+}+2\right], 365[\mathrm{M}+1]^{+}$; Anal. Calcd for $\mathrm{C}_{21} \mathrm{H}_{20} \mathrm{~N}_{2} \mathrm{O}_{4}$ : $\mathrm{C}(69.22 \%), \mathrm{H}(5.53 \%), \mathrm{N}(7.69 \%)$; Found: C(69.34\%), $\mathrm{H}(5.61 \%), \mathrm{N}(7.75 \%)$. 
2.5b Ethyl 2-acetyl-3-[4-(1-methyl-1H-benzimidazol2-ylmethoxy)-phenyl]-acrylate (compound $\mathbf{7 b}$, scheme 3): Yield: $20 \%$. Mp: $145-148^{\circ} \mathrm{C}$; FTIR (KBr): 2960, 2851, 1714, 1695, 1258, 1174, 1103, $799 \mathrm{~cm}^{-1} ;{ }^{1} \mathrm{HNMR}$ $\left(\mathrm{CDCl}_{3}\right)$ mixture of geometric isomers, $\delta: 7.75(\mathrm{~m}$, $1 \mathrm{H}+1 \mathrm{H}), 7.51(\mathrm{~s}, 1 \mathrm{H}), 7.41(\mathrm{~s}, 1 \mathrm{H}), 7.35$ (superimposed $\mathrm{d}, J=8.6 \mathrm{~Hz}, 2 \mathrm{H}+2 \mathrm{H}), 7.28(\mathrm{~m}, 3 \mathrm{H}), 7.03$ (overlapped $\mathrm{d}, J=9.1 \mathrm{~Hz}, 2 \mathrm{H}+2 \mathrm{H}), 5.41(\mathrm{~s}, 2 \mathrm{H}), 5.40(\mathrm{~s}, 2 \mathrm{H}), 4.27$ $(\mathrm{q}, J=7.1 \mathrm{~Hz}, 2 \mathrm{H}), 4.23(\mathrm{q}, J=7.1 \mathrm{~Hz}, 2 \mathrm{H}), 3.86$ (s, 3H), 3.84 (s, 3H), 2.32 (s, 3H), 2.30 (s, 3H), 1.25 $(\mathrm{t}, J=7.1 \mathrm{~Hz}, 3 \mathrm{H}), 1.21(\mathrm{t}, J=7.1 \mathrm{~Hz}, 3 \mathrm{H})$; LCMS $(\mathrm{m} / \mathrm{z}): 379[\mathrm{M}+1]^{+}$; Anal. Calcd for $\mathrm{C}_{22} \mathrm{H}_{22} \mathrm{~N}_{2} \mathrm{O}_{4}$ : C(69.83\%), H(5.87\%); Found: C(69.45\%), H(6.09\%).

2.5c Methyl-2-\{4-[(1-methyl-1H-indol-2-yl)methoxy] benzylidene)-3-oxobutanoate (compound $8 a$, scheme 3): Yield: $15.3 \%$. Mp: $142-144^{\circ} \mathrm{C}$; FTIR (KBr): 2922 and 2851, 1735, 1638, 1240, 1043, $800 \mathrm{~cm}^{-1} ;{ }^{1} \mathrm{H}$ NMR $\left(\mathrm{CDCl}_{3}\right)$ mixture of $E$ and $Z$ isomers, $\delta: 7.63$ (s, $1 \mathrm{H}+1 \mathrm{H}), 7.61(\mathrm{~d}, J=8.0 \mathrm{~Hz}, 1 \mathrm{H}+1 \mathrm{H}), 7.37(\mathrm{~d}, J=$ $8.8 \mathrm{~Hz}, 2 \mathrm{H}+2 \mathrm{H}), 7.34(\mathrm{~m}, 1 \mathrm{H}+1 \mathrm{H}), 7.25(\mathrm{~m}, 1 \mathrm{H}+1 \mathrm{H})$, $7.11(\mathrm{~m}, 1 \mathrm{H}+1 \mathrm{H}), 7.01(\mathrm{~d}, J=8.8 \mathrm{~Hz}, 2 \mathrm{H}+2 \mathrm{H}), 6.61$ $(\mathrm{s}, 1 \mathrm{H}+1 \mathrm{H}), 5.23(\mathrm{~s}, 2 \mathrm{H}), 5.22(\mathrm{~s}, 2 \mathrm{H}), 3.83(\mathrm{~s}, 3 \mathrm{H})$, $3.82(\mathrm{~s}, 3 \mathrm{H}), 3.79$ (superimposed s, 3H+3H), 2.38 (s, $3 \mathrm{H}), 2.36$ (s, 3H); LCMS (m/z): $386\left[\mathrm{M}^{+}+23\right], 365$ $\left[\mathrm{M}^{+}+2\right], 364[\mathrm{M}+1]^{+}$; Anal. Calcd for $\mathrm{C}_{22} \mathrm{H}_{21} \mathrm{NO}_{4}$ : $\mathrm{C}(72.71 \%), \mathrm{H}(5.82 \%), \mathrm{N}(3.85 \%)$; Found: $\mathrm{C}(72.63 \%)$, $\mathrm{H}(5.57 \%), \mathrm{N}(3.68 \%)$.

2.5d Ethyl 2-acetyl-3-[4-(1-methyl-1H-indol-2-ylmethoxy)phenyl]-acrylate (compound $8 \boldsymbol{b}$, scheme 3): Yield: 22.5\%. Mp: $128-131^{\circ} \mathrm{C}$ FTIR (KBr): 2983, 2852, 1731, $1715,1242,1145,1108,859 \mathrm{~cm}^{-1} ;{ }^{1} \mathrm{HNMR}\left(\mathrm{CDCl}_{3}\right)$ mixture of geometric isomers, $\delta: 7.55(\mathrm{~s}, 1 \mathrm{H}), 7.53$ $(\mathrm{s}, 1 \mathrm{H}), 7.43(\mathrm{~m}, 1 \mathrm{H}+1 \mathrm{H}), 7.40(\mathrm{~d}, 1 \mathrm{H}+1 \mathrm{H}), 7.31(\mathrm{~d}$, $J=8.8 \mathrm{~Hz}, 2 \mathrm{H}), 7.27(\mathrm{~d}, J=8.5 \mathrm{~Hz}, 2 \mathrm{H}), 7.05(\mathrm{~m}$, $1 \mathrm{H}+1 \mathrm{H}), 6.96(\mathrm{~m}, 1 \mathrm{H}+1 \mathrm{H}), 6.94$ (overlapped d, $J=$ $8.8 \mathrm{~Hz}, 2 \mathrm{H}+2 \mathrm{H}, \mathrm{Ar}$ ), 6.54 (superimposed s, $1 \mathrm{H}+1 \mathrm{H}$, Ar), 5.16 (s, 2H), 5.15 (s, 2H), 4.29 (q, $J=7.2 \mathrm{~Hz}$, $2 \mathrm{H}), 4.22(\mathrm{q}, J=7.1 \mathrm{~Hz}, 2 \mathrm{H}), 3.72$ (superimposed $\mathrm{s}$, $3 \mathrm{H}+3 \mathrm{H}), 2.33(\mathrm{~s}, 3 \mathrm{H}), 2.31(\mathrm{~s}, 3 \mathrm{H}), 1.25$ (overlapped t, $J=7.2 \mathrm{~Hz}, 3 \mathrm{H}+3 \mathrm{H}) ; \mathrm{LCMS}(\mathrm{m} / \mathrm{z}): 378[\mathrm{M}+1]^{+}, 364$; Anal. Calcd for $\mathrm{C}_{23} \mathrm{H}_{23} \mathrm{NO}_{4}$ : $\mathrm{C}(73.19 \%), \mathrm{H}(6.14 \%)$, $\mathrm{N}(3.71 \%)$; Found: C(73.44\%), H(6.52\%), N(3.98\%).

2.5e Methyl 2-\{4-[(1-methyl-1H-benzimidazol-2-yl)methoxy] benzyl\}-3-oxobutanoate (compound 9a, scheme 3): Yield: $18.0 \%$. Mp: $135-138^{\circ} \mathrm{C}$; FTIR (KBr): 2918 and 2850, 1730, 1711, 1240, 1067, $846 \mathrm{~cm}^{-1} ;{ }^{1} \mathrm{H}$ NMR $\left(\mathrm{CDCl}_{3}\right) \delta: 7.68(\mathrm{~m}, 1 \mathrm{H}), 7.32(\mathrm{~m}, 3 \mathrm{H}), 6.89(\mathrm{~d}, J=$ $8.68 \mathrm{~Hz}, 2 \mathrm{H}), 6.71(\mathrm{~d}, J=8.60 \mathrm{~Hz}, 2 \mathrm{H}), 5.33(\mathrm{~s}, 2 \mathrm{H})$, $3.87(\mathrm{~s}, 3 \mathrm{H}), 3.76(\mathrm{~s}, 3 \mathrm{H}), 3.64(\mathrm{~d}, J=4.6 \mathrm{~Hz}, 1 \mathrm{H}), 3.27$ $(\mathrm{d}, J=16.88 \mathrm{~Hz}, 1 \mathrm{H}), 3.10(\mathrm{~d}, J=16.92 \mathrm{~Hz}, 1 \mathrm{H}), 2.42$ (s, 3H); LCMS (m/z): $405\left[\mathrm{M}^{+}+39\right], 366[\mathrm{M}]^{+}, 261$; Anal. Calcd for $\mathrm{C}_{21} \mathrm{H}_{22} \mathrm{~N}_{2} \mathrm{O}_{4}$ : $\mathrm{C}(68.84 \%), \mathrm{H}(6.05 \%)$, $\mathrm{N}(7.65 \%)$. Found: C(68.59\%), H(6.23\%), N(7.52\%).

2.5f Ethyl 2-[4-(1-Methyl-1H-benzimidazol-2-ylmethoxy)benzyl]-3-oxo-butyrate (compound 9b, scheme 3): Yield: $20 \%$. Mp: $119-122^{\circ} \mathrm{C}$; FTIR (KBr): 2925, 2853, 1738, 1714, 1461, 1241, 1155, 1112, 1040, $854 \mathrm{~cm}^{-1}$; ${ }^{1} \mathrm{HNMR}\left(\mathrm{CDCl}_{3}\right) \delta: 7.77(\mathrm{~d}, J=7.2 \mathrm{~Hz}, 1 \mathrm{H}), 7.32$ $(\mathrm{m}, 3 \mathrm{H}), 7.08(\mathrm{~d}, J=8.7 \mathrm{~Hz}, 2 \mathrm{H}), 6.97(\mathrm{~d}, J=$ $8.7 \mathrm{~Hz}, 2 \mathrm{H}), 5.35(\mathrm{~s}, 2 \mathrm{H}), 4.10(\mathrm{q}, J=7.2 \mathrm{~Hz}, 2 \mathrm{H})$, $3.88(\mathrm{~s}, 3 \mathrm{H}), 3.73(\mathrm{t}, J=7.6 \mathrm{~Hz} 1 \mathrm{H}), 3.07(\mathrm{~d}, J=$ $7.6 \mathrm{~Hz}, 2 \mathrm{H}), 2.29$ (s, 3H), $1.17(\mathrm{t}, J=7.20 \mathrm{~Hz}, 3 \mathrm{H})$; LCMS (m/z): $404\left[\mathrm{M}^{+}+23\right], 381[\mathrm{M}+1]^{+}, 380[\mathrm{M}]^{+}$; Anal. Calcd for $\mathrm{C}_{22} \mathrm{H}_{24} \mathrm{~N}_{2} \mathrm{O}_{4}$ : $\mathrm{C}(69.46 \%), \mathrm{H}(6.36 \%)$, N(7.36\%); Found: C(69.91\%), H(6.74\%), N(6.95\%).

2.5g Methyl 2-\{4-[(1-methyl-1H-indol-2-yl)methoxy] benzyl\}-3-oxobutanoate (compound 10a, scheme 3): Yield: $14.5 \%$. Mp: $122-124^{\circ} \mathrm{C}$; FTIR (KBr): 2917 and 2849, 1730, 1637, 1216, 1103, $847 \mathrm{~cm}^{-1} ;{ }^{1} \mathrm{H}$ NMR $\left(\mathrm{CDCl}_{3}\right) \delta: 7.44(\mathrm{~d}, J=8.4 \mathrm{~Hz}, 1 \mathrm{H}), 7.37(\mathrm{~d}, J=$ $8.8 \mathrm{~Hz}, 2 \mathrm{H}), 7.20(\mathrm{~m}, 2 \mathrm{H}), 7.11(\mathrm{~m}, 1 \mathrm{H}), 6.93(\mathrm{~d}, J=$ $8.8 \mathrm{~Hz}, 2 \mathrm{H}), 6.68(\mathrm{~s}, 1 \mathrm{H}), 5.26(\mathrm{~s}, 2 \mathrm{H}), 3.86(\mathrm{~s}, 3 \mathrm{H}), 3.78$ $(\mathrm{t}, J=7.0 \mathrm{~Hz}, 1 \mathrm{H}), 3.63(\mathrm{~s}, 3 \mathrm{H}), 3.48(\mathrm{~d}, J=7.2 \mathrm{~Hz}$, 2H), 2.28 (s, 3H); LCMS (m/z): $365[\mathrm{M}]^{+}, 322,285$; Anal. Calcd for $\mathrm{C}_{22} \mathrm{H}_{23} \mathrm{NO}_{4}$ : $\mathrm{C}(72.31 \%), \mathrm{H}(6.34 \%)$, $\mathrm{N}(3.83 \%)$; Found: C(72.49\%), H(6.51\%), N(3.72\%).

2.5h Ethyl 2-[4-(1-methyl-1H-indol-2-ylmethoxy)benzyl]-3-oxo-butyrate (compound 10b, scheme 3): Yield: $18 \%$. Mp: $107-110^{\circ} \mathrm{C}$; FTIR (KBr): 2923, 2852 , 1740, 1714, 1235, 1177, 1075, $823 \mathrm{~cm}^{-1} ;{ }^{1} \mathrm{HNMR}$ $\left(\mathrm{CDCl}_{3}\right) \delta: 7.60(\mathrm{~d}, J=7.8 \mathrm{~Hz}, 1 \mathrm{H}), 7.10(\mathrm{~m}, 1 \mathrm{H}), 7.33$ $(\mathrm{m}, 1 \mathrm{H}), 7.07(\mathrm{~d}, J=8.6 \mathrm{~Hz}, 2 \mathrm{H}), 6.91(\mathrm{~d}, J=8.6 \mathrm{~Hz}$, $2 \mathrm{H}), 5.15(\mathrm{~s}, 2 \mathrm{H}), 4.13(\mathrm{q}, J=7.1 \mathrm{~Hz}, 2 \mathrm{H}), 3.79(\mathrm{~s}$, $3 \mathrm{H}), 3.75(\mathrm{~m}, 1 \mathrm{H}), 3.09(\mathrm{~d}, J=7.5 \mathrm{~Hz}, 2 \mathrm{H}), 2.30$ (s. $3 \mathrm{H}), 1.20(\mathrm{t}, J=7.2 \mathrm{~Hz}, 3 \mathrm{H})$; LCMS (m/z): 380 $[\mathrm{M}+1]^{+}, 379[\mathrm{M}]^{+}, 333$; Anal. Calcd for $\mathrm{C}_{23} \mathrm{H}_{25} \mathrm{NO}_{4}$ : $\mathrm{C}(72.80 \%), \mathrm{H}(6.64 \%), \mathrm{N}(3.69 \%)$; Found: $\mathrm{C}(73.15 \%)$, $\mathrm{H}(6.83 \%), \mathrm{N}(4.01 \%)$.

\subsection{Synthetic procedures for the preparation} of the final $\alpha$-alkoxy propanoic acid based NCEs (compounds $14 a$ and $14 b$ )

2.6a General procedure for the syntheses of $11 \mathrm{a}$ and $11 \mathrm{~b}$ : To a slurry of (methoxymethyl)triphenylphosphonium chloride $(2.7 \mathrm{~g}, 0.008 \mathrm{~mol})$ and diisopropylamine $(0.76 \mathrm{~mL}, 0.006 \mathrm{~mol})$ in THF $(20 \mathrm{~mL})$ 
was added a $2.5 \mathrm{M} n$-butyllithium solution in hexane $(2.6 \mathrm{~mL}, 6 \mathrm{mmol})$, at $-10^{\circ} \mathrm{C}$. After $1 \mathrm{~h}$ at $-10^{\circ} \mathrm{C}$ a solution of $\mathbf{1 a} / \mathbf{2} \mathbf{b}(4 \mathrm{mmol})$ in THF $(4 \mathrm{~mL})$ was added. The mixture was allowed to warm to rt over $2 \mathrm{~h}$, then poured into water $(20 \mathrm{~mL})$, and extracted with ether $(3$ $\times 20 \mathrm{~mL}$ ). The combined extracts were washed with brine, dried over sodium sulphate, and concentrated. The required product 11a/b (1:2 mixture of geometrical isomers) was isolated as a white solid by column chromatography using a mixture of hexane and ethyl acetate.

$2.6 b \quad 2-(\{4-[2-M e t h o x y e t h e n y l] p h e n o x y\} m e t h y l)-1-$ methyl-1H-benzimidazole (compound 11a, scheme 4): Yield: $34.2 \%$. Mp: $101{ }^{\circ} \mathrm{C}$; FTIR (KBr):3033, 2930 and 2860, 1653, 1243, 1094, 766, $670 \mathrm{~cm}^{-1} ;{ }^{1} \mathrm{H}$ NMR $\left(\mathrm{CDCl}_{3}\right) \delta$ : mixture of geometrical isomers, $7.84(\mathrm{~m}$, $2 \mathrm{H}), 7.50(\mathrm{~d}, J=7.0 \mathrm{~Hz}, 2 \mathrm{H}), 7.33(\mathrm{~m}, 6 \mathrm{H}), 7.15$ $(\mathrm{d}, J=7.0 \mathrm{~Hz}, 2 \mathrm{H}), 7.00(\mathrm{~d}, J=7.0 \mathrm{~Hz}, 2 \mathrm{H}), 6.91$ $(\mathrm{d}, J=13.0 \mathrm{~Hz}, 1 \mathrm{H}), 6.05(\mathrm{~d}, J=7.0 \mathrm{~Hz}, 1 \mathrm{H}), 5.75$ $(\mathrm{d}, J=13.0 \mathrm{~Hz}, 1 \mathrm{H}), 5.43(\mathrm{~s}, 2 \mathrm{H}), 5.41(\mathrm{~s}, 2 \mathrm{H}) 5.15$ $(\mathrm{d}, J=7.0 \mathrm{~Hz}, 1 \mathrm{H}), 3.90$ (s, 3H), 3.89 (s, 3H), 3.74 (s, 3H), $3.65(\mathrm{~s}, 3 \mathrm{H})$; LCMS (m/z): $295[\mathrm{M}+1]^{+}$; Anal. Calcd for $\mathrm{C}_{18} \mathrm{H}_{18} \mathrm{~N}_{2} \mathrm{O}_{2}$ : $\mathrm{C}(73.45 \%), \mathrm{H}(6.16 \%)$, $\mathrm{N}(9.52 \%)$; Found: C(73.24\%), H(6.35\%), N(9.26\%).

$2.6 \mathrm{c}$ 2-(\{4-[2-Methoxyethenyl]phenoxy\}methyl)-1methyl-1H-indole (compound 11b, scheme 4): Yield: 32.0\%. Mp: 108-109 ${ }^{\circ}$; FTIR (KBr): 3035, 2932 and $2800,1645,1233,1114,752,635 \mathrm{~cm}^{-1} ;{ }^{1} \mathrm{H}$ NMR $\left(\mathrm{CDCl}_{3}\right) \delta$ : mixture of geometrical isomers, 7.67 $(\mathrm{m}, 2 \mathrm{H}), 7.61(\mathrm{~d}, 2 \mathrm{H}), 7.54(\mathrm{~d}, J=8.2 \mathrm{~Hz}, 2 \mathrm{H}), 7.53$ $(\mathrm{m}, 1 \mathrm{H}), 7.32(\mathrm{~d}, J=8.2 \mathrm{~Hz}, 2 \mathrm{H}), 7.23(\mathrm{~m}, 2 \mathrm{H}), 7.21$ $(\mathrm{d}, J=8.8 \mathrm{~Hz}, 2 \mathrm{H}), 7.16(\mathrm{~m}, 1 \mathrm{H}), 7.08(\mathrm{~d}, J=8.8 \mathrm{~Hz}$, $2 \mathrm{H}), 6.94(\mathrm{~d}, J=13.0 \mathrm{~Hz}, 1 \mathrm{H}), 6.58$ (superimposed s, 2H), $6.06(\mathrm{~d}, J=7.0 \mathrm{~Hz}, 1 \mathrm{H}), 5.78(\mathrm{~d}, J=13,0 \mathrm{~Hz}$,
1H), $5.16(\mathrm{~s}, 2 \mathrm{H}), 5.17(\mathrm{~s}, 2 \mathrm{H}), 3.80(\mathrm{~d}, J=7.0 \mathrm{~Hz}$, $1 \mathrm{H}), 3.80(\mathrm{~s}, 3 \mathrm{H}), 3.79(\mathrm{~s}, 3 \mathrm{H}), 3.75(\mathrm{~s}, 3 \mathrm{H}), 3.66$ (s, 3H); LCMS (m/z): $294(\mathrm{M}+1)$; Anal. Calcd for $\mathrm{C}_{19} \mathrm{H}_{19} \mathrm{NO}_{2}$ : $\mathrm{C}(77.79 \%), \mathrm{H}(6.53 \%), \mathrm{N}(4.77 \%)$; Found: $\mathrm{C}(77.24 \%), \mathrm{H}(6.87 \%), \mathrm{N}(5.26 \%)$.

2.6d General procedure for the syntheses of the acetals $12 a$ and 12b: A solution 11a/b $(2.5 \mathrm{mmol})$ and $p$-toluenesulphonic acid monohydrate $(0.026 \mathrm{mmol})$ in iso-Butyl alcohol $(7 \mathrm{~mL})$ was heated to reflux overnight. The solvent was removed, the residue was taken up in ethyl acetate, and the solution was washed with $5 \%$ sodium bicarbonate and brine, dried over sodium sulphate, and concentrated to get $\mathbf{1 2 a} / \mathbf{b}$ as yellow viscous oil which slowly solidified on standing.

2.6e 2-[4-(2,2-Diisobutoxy-ethyl)-phenoxymethyl]-1methyl-1H-benzimidazole (compound 12a, scheme 4): Yield: $65.0 \%$. Mp: $110^{\circ} \mathrm{C}$; FTIR (KBr): 3053, 2956 and $2872,1253,1234,1064,1039,743,570 \mathrm{~cm}^{1} ;{ }^{1} \mathrm{H}$ NMR $\left(\mathrm{CDCl}_{3}\right) \delta: 7.77(\mathrm{~d}, 1 \mathrm{H}), 7.31(\mathrm{~m}, 3 \mathrm{H}), 7.16(\mathrm{~d}$, $J=8.6 \mathrm{~Hz}, 2 \mathrm{H}), 6.98(\mathrm{~d}, J=8.6 \mathrm{~Hz}, 2 \mathrm{H}) 5.35(\mathrm{~s}$, $2 \mathrm{H}), 4.53(\mathrm{t}, 1 \mathrm{H}), 3.86(\mathrm{~s}, 3 \mathrm{H}), 3.37(\mathrm{dd}, J=6.5 \mathrm{~Hz}$ and $9.1 \mathrm{~Hz}, 2 \mathrm{H}), 3.13(\mathrm{dd}, J=6.5 \mathrm{~Hz}$ and $9.06 \mathrm{~Hz}$, $2 \mathrm{H}), 2.84(\mathrm{~d}, 2 \mathrm{H}), 1.78(\mathrm{~m}, 2 \mathrm{H}), 0.86(\mathrm{~d}, 6 \mathrm{H}), 0.84$ $(\mathrm{d}, 6 \mathrm{H})$; LCMS (m/z): $411[\mathrm{M}+1]^{+}$; Anal. Calcd for $\mathrm{C}_{25} \mathrm{H}_{34} \mathrm{~N}_{2} \mathrm{O}_{3}$ : $\mathrm{C}(73.14 \%), \mathrm{H}(8.35 \%), \mathrm{N}(6.82 \%)$; Found: $\mathrm{C}(73.55 \%), \mathrm{H}(8.12 \%), \mathrm{N}(6.51 \%)$.

$2.6 \mathrm{f}$ 2-[4-(2, 2-Diisobutoxy-ethyl)-phenoxymethyl]-1methyl-1H-indole (compound 12b, scheme 4): Yield: 65\%. Mp: $120-122^{\circ} \mathrm{C}$; FTIR (KBr): 3062, 2948 and 2862, 1246, 1231, 1116, 753, $638 \mathrm{~cm}^{1} ;{ }^{1} \mathrm{H}$ NMR $\left(\mathrm{CDCl}_{3}\right) \delta: 7.51(\mathrm{~d}, 1 \mathrm{H}), 7.24(\mathrm{~d}, 1 \mathrm{H}), 7.14(\mathrm{~m}, 2 \mathrm{H})$, $7.03(\mathrm{~d}, J=8.6 \mathrm{~Hz}, 2 \mathrm{H}), 6.67(\mathrm{~d}, J=8.6 \mathrm{~Hz}, 2 \mathrm{H}), 6.38$ $(\mathrm{s}, 1 \mathrm{H}), 4.58(\mathrm{~s}, 2 \mathrm{H}), 4.47(\mathrm{t}, J=5.6 \mathrm{~Hz}, 1 \mathrm{H}), 3.71(\mathrm{~s}$,

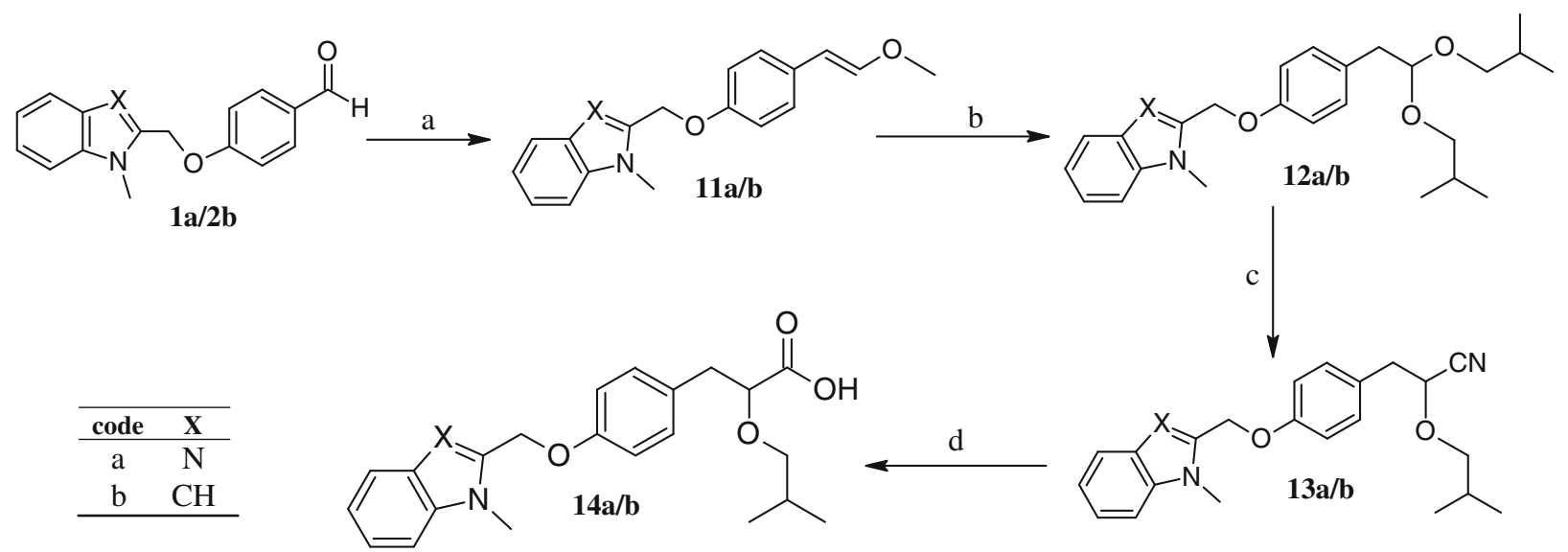

Scheme 4. Reagents and conditions: (a) $\mathrm{Ph}_{3} \mathrm{P}^{+} \mathrm{CH}_{2} \mathrm{OMe} \mathrm{Cl}^{-}, \mathrm{LDA}, \mathrm{THF},-10^{\circ} \mathrm{C}$; (b) isobutanol; (c) $\mathrm{TMSCN}$, $\mathrm{BF}_{3}-\mathrm{DCM}$; (d) $\mathrm{NaOH}, \mathrm{H}_{2} \mathrm{O}-\mathrm{EtOH}$. 
$3 \mathrm{H}), 3.31(\mathrm{dd}, J=6.5 \mathrm{~Hz}$ and $9.1 \mathrm{~Hz}, 2 \mathrm{H}), 3.07$ $(\mathrm{dd}, J=6.5 \mathrm{~Hz}$ and $9.1 \mathrm{~Hz}, 2 \mathrm{H}), 2.77(\mathrm{~d}, 2 \mathrm{H}), 1.71$ $(\mathrm{m}, 2 \mathrm{H}), 1.18(\mathrm{~d}, 12 \mathrm{H})$; LCMS $(\mathrm{m} / \mathrm{z}): 410[\mathrm{M}+1]^{+}$; Anal. Calcd for $\mathrm{C}_{26} \mathrm{H}_{35} \mathrm{NO}_{3}$ : $\mathrm{C}(76.25 \%), \mathrm{H}(8.61 \%)$, $\mathrm{N}(3.42 \%)$; Found: C(76.55\%), H(8.37\%), N(3.51\%).

2.6g General procedure for the syntheses of the cyanides 13a and 13b: To a solution of $12 \mathbf{a} / \mathbf{b}$ $(0.3 \mathrm{mmol})$ in dichloromethane $(8 \mathrm{~mL})$ were added trimethylsilyl cyanide $(0.11 \mathrm{~mL}, 0.9 \mathrm{mmol})$ and boron trifluoride etherate $(0.008 \mathrm{~g}, 0.075 \mathrm{mmol})$. After $1 \mathrm{~h}$ the solution was diluted with dichloromethane, washed with 5\% sodium bicarbonate, water, and brine, dried over sodium sulphate, and concentrated. The crude product was purified by column chromatography eluting with a mixture of hexane and ethyl acetate and $\mathbf{1 3 a} / \mathbf{b}$ was isolated as an off white solid.

2.6h 2-Isobutoxy-3-[4-(1-methyl-1H-benzimidazole2-ylmethoxy)-phenyl]-propionitrile (compound 13a, scheme 4): Yield: $33.0 \%$. Mp: $115-117^{\circ} \mathrm{C}$; FTIR (KBr): 2900, 2800, 2300, 1600, 1240, 1026, 900, $744 \mathrm{~cm}^{-1} ;{ }^{1} \mathrm{H}$ NMR $\left(\mathrm{CDCl}_{3}\right) \delta: 7.85(\mathrm{~d}, 1 \mathrm{H}), 7.40(\mathrm{~m}$, $3 \mathrm{H}), 7.21(\mathrm{~d}, J=8.6 \mathrm{~Hz}, 2 \mathrm{H}), 7.06(\mathrm{~d}, J=8.6 \mathrm{~Hz}$, $2 \mathrm{H}), 5.53(\mathrm{~s}, 2 \mathrm{H}), 4.18(\mathrm{t}, 1 \mathrm{H}), 3.96(\mathrm{~s}, 3 \mathrm{H}), 3.49(\mathrm{dd}$, $J=8.6$ and $6.6 \mathrm{~Hz}), 3.17(\mathrm{dd}, J=8.6 \mathrm{~Hz}$ and $6.5 \mathrm{~Hz}$, $2 \mathrm{H}$ ), 3.05 (overlapped dd, $2 \mathrm{H}, J=6.7$ and 2.9), 1.86 $(\mathrm{m}, 1 \mathrm{H}), 0.86(\mathrm{~d}, 6 \mathrm{H})$; LCMS (m/z): $364[\mathrm{M}+1]^{+}$; Anal. Calcd for $\mathrm{C}_{22} \mathrm{H}_{25} \mathrm{~N}_{3} \mathrm{O}_{2}$ : C(72.70\%), $\mathrm{H}(6.93 \%)$, $\mathrm{N}(11.56 \%)$; Found: C(72.48\%), H(7.23\%), N(11.22\%).

$2.6 \mathrm{i}$ 2-Isobutoxy-3-[4-(1-methyl-1H-indole-2-ylmethoxy)phenyl]-propionitrile (compound 13b, scheme 4): Yield: $31.0 \%$. Mp: $130-132^{\circ} \mathrm{C}$; FTIR (KBr): 2954, 2873, 2322, 1612, 1253, 1032, 922, $749 \mathrm{~cm}^{-1} ;{ }^{1} \mathrm{H}$ NMR $\left(\mathrm{CDCl}_{3}\right) \delta: 7.58(\mathrm{~d}, 1 \mathrm{H}), 7.48(\mathrm{~m}, 2 \mathrm{H}), 7.32(\mathrm{~d}, 2 \mathrm{H})$, $7.22(\mathrm{~d}, J=8.1 \mathrm{~Hz}, 2 \mathrm{H}), 7.10(\mathrm{~d}, J=7.9 \mathrm{~Hz}, 2 \mathrm{H})$, $6.45(\mathrm{~s}, 1 \mathrm{H}), 4.82(\mathrm{~s}, 2 \mathrm{H}), 4.18(\mathrm{t}, J=6.8,1 \mathrm{H}), 3.81$ $(\mathrm{s}, 3 \mathrm{H}), 3.49(\mathrm{dd}, J=8.6$ and $6.6 \mathrm{~Hz}, 1 \mathrm{H}), 3.17(\mathrm{dd}$, $J=8.6 \mathrm{~Hz}$ and $6.5 \mathrm{~Hz}, 1 \mathrm{H}$ ), 3.05 (overlapping dd, $J=$ 6.7 and $2.9,2 \mathrm{H}), 1.85(\mathrm{~m}, 1 \mathrm{H}), 0.87(\mathrm{~d}, 6 \mathrm{H})$; LCMS $(\mathrm{m} / \mathrm{z}): 364[\mathrm{M}+2]^{+}$; Anal. Calcd for $\mathrm{C}_{23} \mathrm{H}_{26} \mathrm{~N}_{2} \mathrm{O}_{2}$ : $\mathrm{C}(76.21 \%), \mathrm{H}(7.23 \%), \mathrm{N}(7.73 \%)$; Found: $\mathrm{C}(75.88 \%)$, $\mathrm{H}(7.35 \%), \mathrm{N}(8.12 \%)$.

2.6j General procedure for the syntheses of the acids $14 a$ and 14b: A mixture of $13 a / b \quad(0.09 \mathrm{mmol})$, ethanol $(7 \mathrm{~mL})$, and $6 \mathrm{~N}$ sodium hydroxide $(0.5 \mathrm{~mL})$ was heated to reflux for $3 \mathrm{~h}$. Water $(2 \mathrm{~mL})$ was added, and the solution was acidified with concentrated hydrochloric acid $(6 \mathrm{~mL})$ and then extracted with ethyl acetate $(2 \times 7 \mathrm{~mL})$. The combined organic layers were washed with brine, dried over sodium sulphate, and concentrated. The crude product was recrystallized from ethyl acetate/hexane and to obtain $14 a / b$ as a white solid.

2.6k 2-Isobutoxy-3-[4-(1-methyl-1H-benzimidazole-2ylmethoxy)-phenyl]-propionic acid: (compound 14a, scheme 4): Yield: $70.0 \%$. Mp: $167-168^{\circ} \mathrm{C}$; FTIR (KBr): 3400, 2900, 2800, 1655, 1238, 1050, 825 $765 \mathrm{~cm}^{-1} ;{ }^{1} \mathrm{H}$ NMR (DMSO-d 6 ) $\delta: 7.63(\mathrm{~d}, 1 \mathrm{H}), 7.56$ (d, 1H), $7.27(\mathrm{t}, 1 \mathrm{H}), 7.20(\mathrm{t}, 1 \mathrm{H}), 7.13(\mathrm{~d}, J=6.8 \mathrm{~Hz}$, $2 \mathrm{H}), 7.00(\mathrm{~d}, J=7.4 \mathrm{~Hz}, 2 \mathrm{H}) 5.27(\mathrm{~s}, 2 \mathrm{H}), 3.83(\mathrm{~s}$, $3 \mathrm{H}), 3.71(\mathrm{t}, 1 \mathrm{H}), 3.37(\mathrm{dd}, J=6.5 \mathrm{~Hz}$ and $9.1 \mathrm{~Hz}$, 2H), $2.84(\mathrm{~d}, 2 \mathrm{H}), 1.6848(\mathrm{~m}, 1 \mathrm{H}), 0.72(\mathrm{~d}, 6 \mathrm{H})$; LCMS $(\mathrm{m} / \mathrm{z})$ : $383[\mathrm{M}+1]^{+}$; Anal. Calcd for $\mathrm{C}_{22} \mathrm{H}_{26} \mathrm{~N}_{2} \mathrm{O}_{4}$ : $\mathrm{C}(69.09 \%), \mathrm{H}(6.85 \%), \mathrm{N}(7.32 \%)$; Found: $\mathrm{C}(69.33 \%)$, $\mathrm{H}(6.56 \%), \mathrm{N}(7.50 \%)$.

2.61 2-Isobutoxy-3-[4-(1-methyl-1H-indole-2-ylmethoxy)phenyl]-propionic acid: (compound 14b, scheme 4): Yield: 67\%. Mp: $175-177^{\circ} \mathrm{C}$; FTIR (KBr): 3430, 2927 ,

Table 1. Designed NCEs.

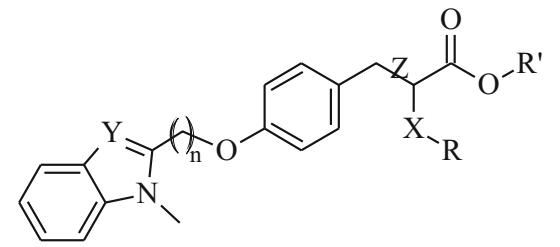

\begin{tabular}{|c|c|c|c|c|c|c|}
\hline $\begin{array}{l}\text { Compound } \\
\text { code }\end{array}$ & $\mathrm{n}$ & Y & $X$ & $\mathrm{R}$ & $\mathrm{R}^{\prime}$ & $Z^{*}$ \\
\hline $7^{\prime} \mathbf{a}$ & 2 & $\mathrm{~N}$ & $\mathrm{C}=\mathrm{O}$ & $\mathrm{Me}$ & $\mathrm{Me}$ & $\mathrm{db}$ \\
\hline $7 \mathbf{a}$ & 1 & $\mathrm{~N}$ & $\mathrm{C}=\mathrm{O}$ & $\mathrm{Me}$ & $\mathrm{Me}$ & $\mathrm{db}$ \\
\hline $7^{\prime} \mathbf{b}$ & 2 & $\mathrm{~N}$ & $\mathrm{C}=\mathrm{O}$ & $\mathrm{Me}$ & Et & $\mathrm{db}$ \\
\hline $7 b$ & 1 & $\mathrm{~N}$ & $\mathrm{C}=\mathrm{O}$ & $\mathrm{Me}$ & Et & $\mathrm{db}$ \\
\hline $8^{\prime} \mathbf{a}$ & 2 & $\mathrm{CH}$ & $\mathrm{C}=\mathrm{O}$ & $\mathrm{Me}$ & $\mathrm{Me}$ & $\mathrm{db}$ \\
\hline $8 \mathbf{a}$ & 1 & $\mathrm{CH}$ & $\mathrm{C}=\mathrm{O}$ & $\mathrm{Me}$ & $\mathrm{Me}$ & $\mathrm{db}$ \\
\hline $8^{\prime} \mathbf{b}$ & 2 & $\mathrm{CH}$ & $\mathrm{C}=\mathrm{O}$ & $\mathrm{Me}$ & Et & $\mathrm{db}$ \\
\hline $8 b$ & 1 & $\mathrm{CH}$ & $\mathrm{C}=\mathrm{O}$ & $\mathrm{Me}$ & Et & $\mathrm{db}$ \\
\hline $9^{\prime} \mathbf{a}$ & 2 & $\mathrm{~N}$ & $\mathrm{C}=\mathrm{O}$ & $\mathrm{Me}$ & $\mathrm{Me}$ & $\mathrm{sb}$ \\
\hline $9 \mathrm{a}$ & 1 & $\mathrm{~N}$ & $\mathrm{C}=\mathrm{O}$ & $\mathrm{Me}$ & $\mathrm{Me}$ & $\mathrm{sb}$ \\
\hline $9^{\prime} \mathbf{b}$ & 2 & $\mathrm{~N}$ & $\mathrm{C}=\mathrm{O}$ & $\mathrm{Me}$ & Et & $\mathrm{sb}$ \\
\hline 9b & 1 & $\mathrm{~N}$ & $\mathrm{C}=\mathrm{O}$ & $\mathrm{Me}$ & Et & $\mathrm{sb}$ \\
\hline $10^{\prime} \mathbf{a}$ & 2 & $\mathrm{CH}$ & $\mathrm{C}=\mathrm{O}$ & $\mathrm{Me}$ & $\mathrm{Me}$ & $\mathrm{sb}$ \\
\hline $10 \mathrm{a}$ & 1 & $\mathrm{CH}$ & $\mathrm{C}=\mathrm{O}$ & $\mathrm{Me}$ & $\mathrm{Me}$ & $\mathrm{sb}$ \\
\hline $10^{\prime} \mathbf{b}$ & 2 & $\mathrm{CH}$ & $\mathrm{C}=\mathrm{O}$ & $\mathrm{Me}$ & Et & $\mathrm{sb}$ \\
\hline 10b & 1 & $\mathrm{CH}$ & $\mathrm{C}=\mathrm{O}$ & $\mathrm{Me}$ & Et & $\mathrm{sb}$ \\
\hline $14^{\prime} \mathrm{a}$ & 2 & $\mathrm{~N}$ & $\mathrm{O}$ & ${ }^{\mathrm{i}} \mathrm{Bu}$ & $\mathrm{H}$ & $\mathrm{sb}$ \\
\hline 14a & 1 & $\mathrm{~N}$ & $\mathrm{O}$ & ${ }^{1} \mathrm{Bu}$ & $\mathrm{H}$ & $\mathrm{sb}$ \\
\hline $14^{\prime} \mathrm{b}$ & 2 & $\mathrm{CH}$ & $\mathrm{O}$ & ${ }^{\mathrm{i}} \mathrm{Bu}$ & $\mathrm{H}$ & $\mathrm{sb}$ \\
\hline 14b & 1 & $\mathrm{CH}$ & $\mathrm{O}$ & ${ }^{\mathrm{i}} \mathrm{Bu}$ & $\mathrm{H}$ & $\mathrm{sb}$ \\
\hline
\end{tabular}

$* \mathrm{db}=$ double bond, $\mathrm{sb}=$ single bond 
2832, 1656, 1240, 1038, 840, $760 \mathrm{~cm}^{-1} ;{ }^{1} \mathrm{H}$ NMR $\left(\right.$ DMSO-d $\left._{6}\right) \delta: 7.65(\mathrm{~d}, 1 \mathrm{H}), 7.48(\mathrm{~d}, 1 \mathrm{H}), 7.30(\mathrm{t}, 2 \mathrm{H})$, $7.15(\mathrm{~d}, J=6.7 \mathrm{~Hz}, 2 \mathrm{H}), 6.99(\mathrm{~d}, J=7.4 \mathrm{~Hz}, 2 \mathrm{H})$, $6.34(\mathrm{~s}, 1 \mathrm{H}), 5.36(\mathrm{~s}, 2 \mathrm{H}), 3.89(\mathrm{~s}, 3 \mathrm{H}), 4.98(\mathrm{t}, J=$ $5.8 \mathrm{~Hz}, 1 \mathrm{H}), 4.27(\mathrm{dd}, J=6.5 \mathrm{~Hz}$ and $9.1 \mathrm{~Hz}, 2 \mathrm{H})$, $2.36(\mathrm{~d}, 2 \mathrm{H}), 1.75(\mathrm{~m}, 1 \mathrm{H}), 0.87(\mathrm{~d}, 6 \mathrm{H})$; LCMS (m/z): $382[\mathrm{M}+1]^{+}$; Anal. Calcd for $\mathrm{C}_{23} \mathrm{H}_{27} \mathrm{NO}_{4}: \mathrm{C}(72.42 \%)$, $\mathrm{H}(7.13 \%), \mathrm{N}(3.67 \%)$; Found: $\mathrm{C}(72.13 \%), \mathrm{H}(6.66 \%)$, $\mathrm{N}(4.15 \%)$.

\subsection{Design of NCEs as PPAR $\alpha / \gamma$ partial agonists and prediction of PPAR $\alpha$ and PPAR $\gamma$ activities}

The novel molecules were designed by thoroughly studying the structural features required for partial agonism to both PPAR $\alpha$ and PPAR $\gamma$ obtained from contour analysis of the developed PPAR $\alpha$ and PPAR $\gamma$ 3D-QSAR CoMFA models. ${ }^{9}$ The critical observation of the structural features of the highly potent dual agonist farglitazar and PPAR $\gamma$ full agonist rosiglitazone also aided to the design of the new molecules (table 1) expected to be $\operatorname{PPAR} \alpha / \gamma$ partial agonists. The designed molecules were modelled onto the ligand,
Farglitazar, extracted from PPAR $\gamma$ crystal structure (PDB code 1FM9) by the 'build molecule' module of Sybyl7.3. ${ }^{16}$ After the energy optimization of the sketched molecules by Powell method (using the default parameters, Gasteiger-Marsili charges have been assigned), they were all subjected to activity prediction by the developed PPAR $\alpha, \operatorname{PPAR} \gamma$ and PPAR dual CoMFA models. ${ }^{9}$ The predicted activities in terms of $\mathrm{pEC}_{50}$ values are listed in table 2. The ketoester molecules in ' $\mathrm{R}$ ' configuration whereas the $\alpha$-alkoxy acid molecules in ' $S$ ' were predicted for identical and optimum alignment (figures 9, 10).

\subsection{Molecular docking studies}

The sketched molecules (some standard dual activators and designed molecules) were docked into ligand binding active site of PPAR $\gamma$ crystal structure by the Surflex Dock method. The surflex dock score (total score) and the $\mathrm{G}$ score value for best docked conformation of each molecule were recorded (table 2). The number of hydrogen bonding interactions of the docked ligands with the active site and the amino acid residues involved

Table 2. Predicted PPAR activities and binding affinities at PPAR $\gamma$ active site of the synthesized NCEs and standard molecules.

\begin{tabular}{|c|c|c|c|c|c|}
\hline Compound $^{\ddagger}$ & Predicted $\alpha \mathrm{pEC}_{50}$ & Predicted $\gamma \mathrm{pEC}_{50}$ & Predicted dual $\mathrm{pEC}_{50}$ & Total score & G-score* \\
\hline Farglitazar & 6.307 & 9.542 & 16.418 & 11.62 & -390.56 \\
\hline LY 510929 & 8.418 & 8.768 & 17.092 & 8.85 & -301.54 \\
\hline GW409544 & 6.125 & 9.491 & 15.348 & 9.35 & -363.39 \\
\hline DRF 2725 & 5.799 & 6.471 & 12.076 & 8.08 & -289.41 \\
\hline GW 7845 & 5.912 & 9.301 & 14.988 & 8.72 & -356.52 \\
\hline NND 9 & 6.230 & 6.609 & 12.797 & 7.06 & -302.76 \\
\hline $7^{\prime} \mathbf{a}$ & 6.176 & 6.727 & 12.857 & 6.46 & -254.12 \\
\hline $7 \mathbf{a}$ & 6.093 & 6.634 & 12.785 & 4.37 & -218.91 \\
\hline $8^{\prime} \mathbf{a}$ & 6.225 & 6.782 & 12.987 & 7.05 & -280.81 \\
\hline $8 \mathbf{a}$ & 6.159 & 6.673 & 12.828 & 5.77 & -221.81 \\
\hline $9^{\prime} \mathbf{a}$ & 6.387 & 7.213 & 13.524 & 5.64 & -271.34 \\
\hline 9a & 6.121 & 6.605 & 12.062 & 4.43 & -242.49 \\
\hline $10^{\prime} \mathbf{a}$ & 6.325 & 7.109 & 13.301 & 5.47 & -278.49 \\
\hline $10 a$ & 6.143 & 6.649 & 12.657 & 4.71 & -260.76 \\
\hline $7^{\prime} \mathbf{b}$ & 6.457 & 7.197 & 13.529 & 5.25 & -208.26 \\
\hline $7 \mathrm{~b}$ & 5.783 & 6.720 & 12.375 & 3.56 & -216.53 \\
\hline $8^{\prime} \mathbf{b}$ & 6.165 & 6.903 & 13.075 & 1.34 & -332.76 \\
\hline $8 b$ & 5.971 & 6.360 & 12.204 & 4.79 & -177.43 \\
\hline $9^{\prime} \mathbf{b}$ & 6.400 & 7.210 & 13.449 & 4.82 & -251.90 \\
\hline 9b & 6.198 & 6.762 & 12.906 & 5.69 & -236.34 \\
\hline $10^{\prime} \mathrm{b}$ & 6.367 & 7.055 & 13.236 & 3.69 & -254.44 \\
\hline $10 \mathrm{~b}$ & 6.114 & 6.402 & 12.397 & 6.71 & -266.91 \\
\hline $14^{\prime} \mathrm{a}$ & 6.706 & 7.519 & 14.029 & 7.88 & -294.91 \\
\hline $14 a$ & 6.135 & 6.528 & 12.470 & 6.93 & -295.49 \\
\hline $14^{\prime} \mathrm{b}$ & 6.490 & 7.189 & 13.400 & 7.25 & -281.33 \\
\hline $14 b$ & 6.275 & 6.568 & 12.643 & 8.06 & -306.42 \\
\hline
\end{tabular}

${ }^{\ddagger}$ The benzyl ketoester molecules are in 'R' configuration and $\alpha$-alkoxy acid molecules in 'S' configuration

*G score of the best docked conformation 
Table 3. The amino acid residues involved in hydrogen bonding interactions with the ligands at the PPAR $\gamma$ active site (1FM9).

\begin{tabular}{lcc}
\hline Compound & Amino acid residues (no. of H-bonding interactions) & Total no. of H-bonds \\
\hline Farglitazar & S 289 (1), H 323 (1), H 449 (1), Y 473 (1) & 4 \\
GW409544 & S 289(1), H 323 (1), H 449(1), Y 473 (1) & 4 \\
LY 510929 & Y 327 (1), H 449 (1), Y 473 (2) & 4 \\
DRF 2725 & S 289 (1), H 449 (1), Y 473 (1) & 3 \\
GW 7845 & S 289(1), H 323 (1), H 449 (1), Y 473 (1) & 4 \\
NND 9 & S 289 (1), H 449 (1), Y 473 (1) & 3 \\
7a & S 342 (1) & 1 \\
7b & S 342 (1) & 1 \\
8a & ARG288 (1) & 1 \\
8b & S 342 (1) & 1 \\
9a & S 289 (1) & 1 \\
9b & S 342 & 1 \\
10a & S 289 (1) & 1 \\
10b & S 289 (1) & 3 \\
14a & S 289 (2), H 323 (1) & 3 \\
14b & Y 473 (2), H 449 (1) & 1 \\
\hline
\end{tabular}

were also found (table 3 ) and compared with that of the standard molecules.

\section{Results and discussion}

$\operatorname{PPAR} \alpha / \gamma$ dual agonists mainly the tyrosine and propanoic acid derivatives usually possess essential pharmacophoric elements ${ }^{10-12}$ i.e.; an acidic group or a hydrogen bonding part attached to a central flat aromatic ring, a linker and a large hydrophobic fragment and adopts a bioactive U-shapped conformation for receptor binding ${ }^{13}$ (figure 2). We have previously reported the development of three 3D-QSAR CoMFA models namely; PPAR $\alpha$, PPAR $\gamma$ and PPAR dual, using a set of known dual agonists (tyrosine and $\alpha$-alkoxy/ aryloxy propanoic acid derivatives) for which in vitro PPAR transactivation assay activities are reported. ${ }^{9}$ From the analysis of the steric and electrostatic contours resulted from the models, a set of molecules expected to be $\operatorname{PPAR} \alpha / \gamma$ partial agonists were designed according

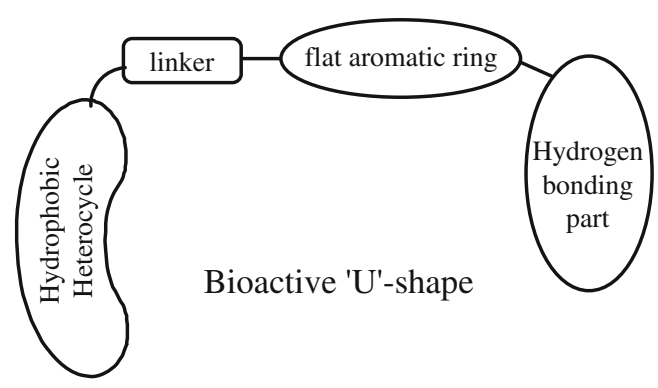

Figure 2. The pharmacophore. to the steric and electronic requirements and were subsequently predicted for their activities (tables 1 and 2). Two benzfused heterocycles namely; benzimidazole and indole, popular for their versatile pharmacological potential, were taken according to design guided by the analysis of the CoMFA contours and chemical structure analysis of the known standard PPAR molecules for the hydrophobic part to reduce the extending length of the hydrophobic unit (figures 3-8) unlike a phenyl oxazole of farglitar or muraglitazar; thiophenyl oxazole of LY 510929; a tricyclic phenoxazine of ragaglitazar or carbazole of NND9 (figures 5 and 6). Fusion of the five and six-membered rings of the hydrophobic part of farglitazar to get a benzfused heterocycle (figure 6) decreases the extending length of the hydrophobic unit (figures 7 and 8). The heterocyclic left hand side part of full agonist rosiglitazone can also be structurally modified by joining the heteroatom of the $N$-methyl pyridyl moiety to the carbon atom adjacent to the $N$-Me group of the spacer to obtain a benzfused heterocycle benzimidazole with one heteroatom substituted with a methyl group (figure 6). The bioisosteric replacement of the unsubstituted nitrogen with a ' $\mathrm{CH}$ ' group gives rise to other benzfused heterocycle indole with the heteroatom substituted with a methyl group (figure 6). Such heterocyclic scaffolds reported here contributed to the reduction of PPAR $\alpha$ and PPAR $\gamma$ activities compared to the previously known molecules. The heteroatom (nitrogen) of the heterocycles with methyl group substitution was desirable to suppress electron richness (red rhombohedral contour on the left hand side in the PPAR $\alpha$ and PPAR $\gamma$ models) simultaneously rendering less bulk in the region (yellow contour on the left hand side of 


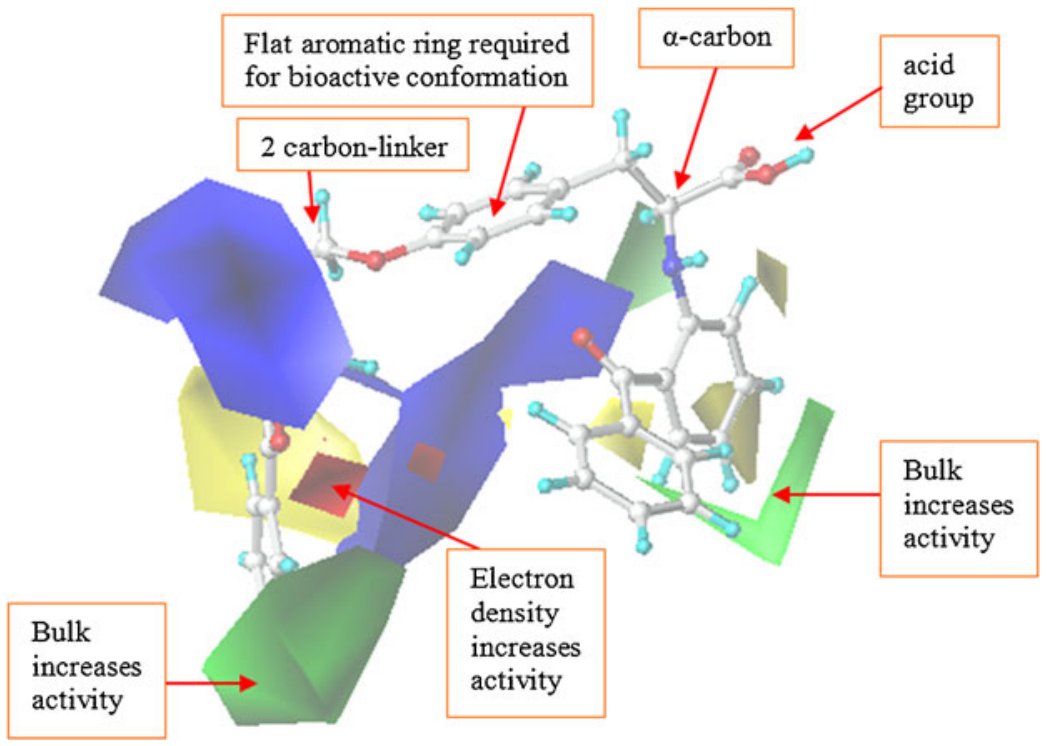

Figure 3. Steric and electrostatic solid contours of the PPAR $\alpha$ model $^{9}$ (Farglitazar shown as the standard ligand, special features for the design of the reported NCEs as partial agonists have been highlighted).

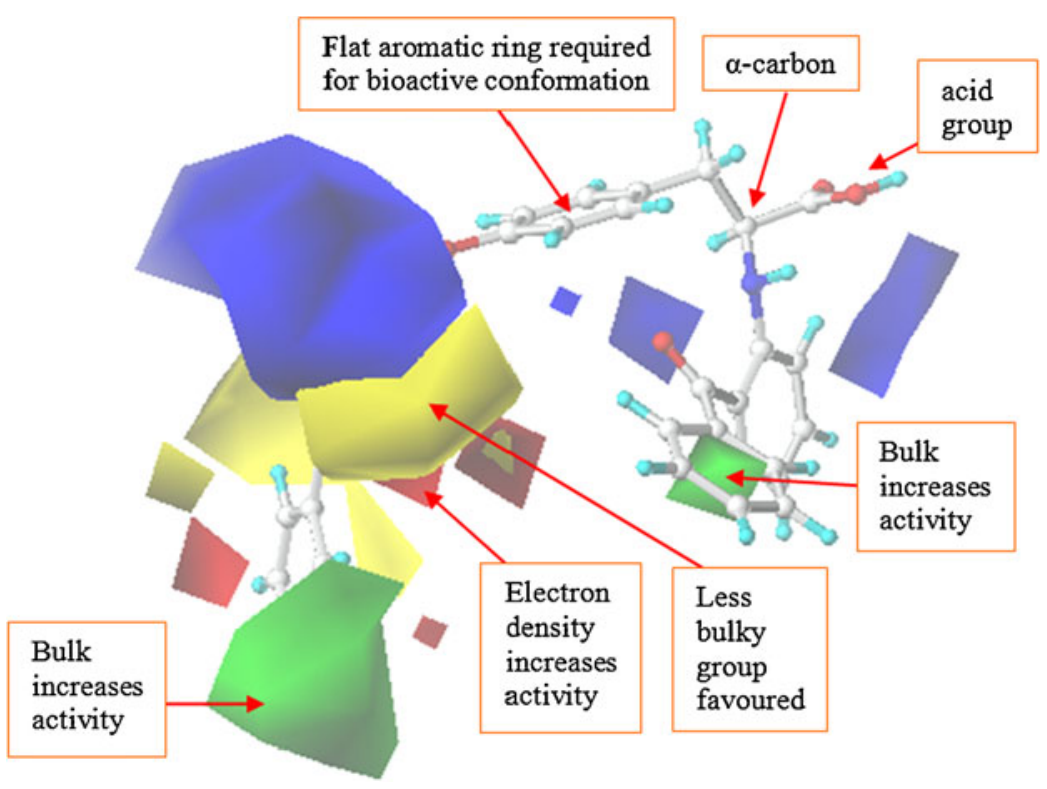

Figure 4. Steric and electrostatic solid contours of the PPAR $\gamma \operatorname{model}^{9}$ (Farglitazar shown as the standard ligand, special features for the design of the reported NCEs as partial agonists have been highlighted).

PPAR $\gamma$ model) as found from observation and analysis of the contours ${ }^{9}$ (figures 3, 4, 7 and 8) and also keeping in view the housability of the ligands at the receptor site. The length of the two carbon linker (as reported in farglitazar, ragaglitazar, tesaglitazar, etc.) was decreased to one carbon to impart further constraint to the designed molecules. Although NCEs with ethyleneoxy as well as methyleneoxy linker were predicted for their PPAR activities and binding affinities and results obtained were as expected and supported that methyleneoxy, linker is preferred over ethyleneoxy for partial agonism (table 2). The effect of longer and shorter spacer (ethyleneoxy and methyleneoxy, respectively) has been shown in figures 9 and 10. It can be observed that the hydrophobic units (left hand side) of the molecules with ethyleneoxy spacer (table 1) enters into the green steric contour which actually favours the increase in activities of the molecules whereas heterocyclic 


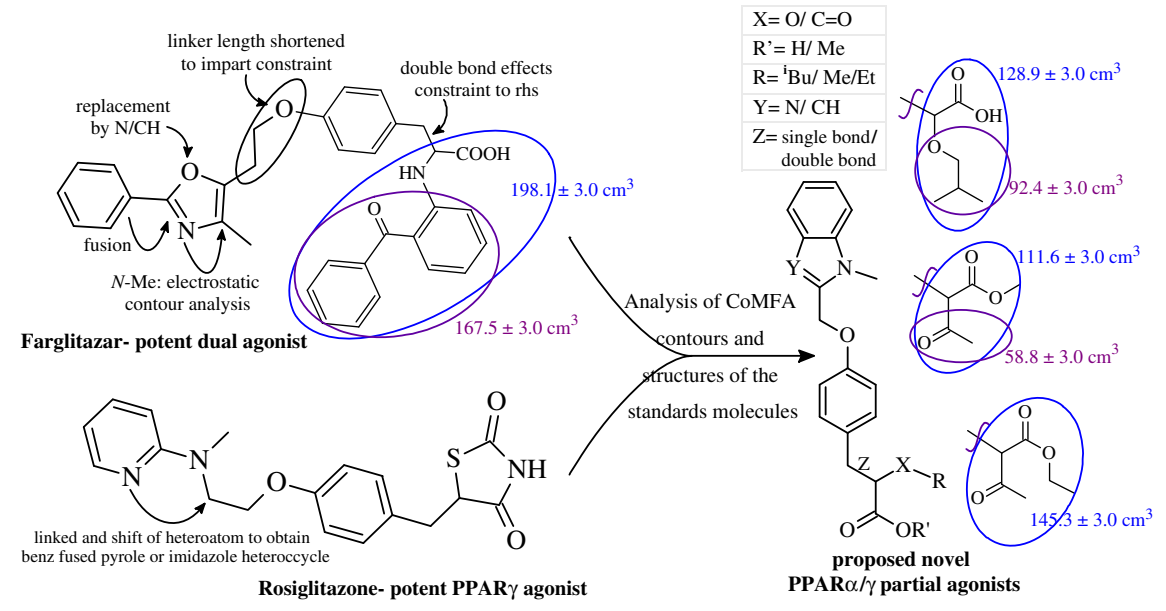

Figure 5. Design of the acyclic analogs of oxazolidenedione and isoxazolidenedione as PPAR $\alpha$ and PPAR $\gamma$ partial agonists: structural modification of the hydrophobic part of the template Farglitazar (highly potent dual agonist) and Rosiglitazone (highly potent PPAR $\gamma$ agonist) to reach to the conclusion of introducing benzfused imidazole or pyrole (also guided and supported by CoMFA steric and electrostatic contours) as the hydrophobic units in the design of partial PPAR $\alpha / \gamma$ activators. The effect of reducing the size by incorporating alkoxy/alkanoyl group on the total volume of the hydrogen bonding part is also shown. (The numbers in blue and violet indicate calculated molar volume of the respective fragment.)

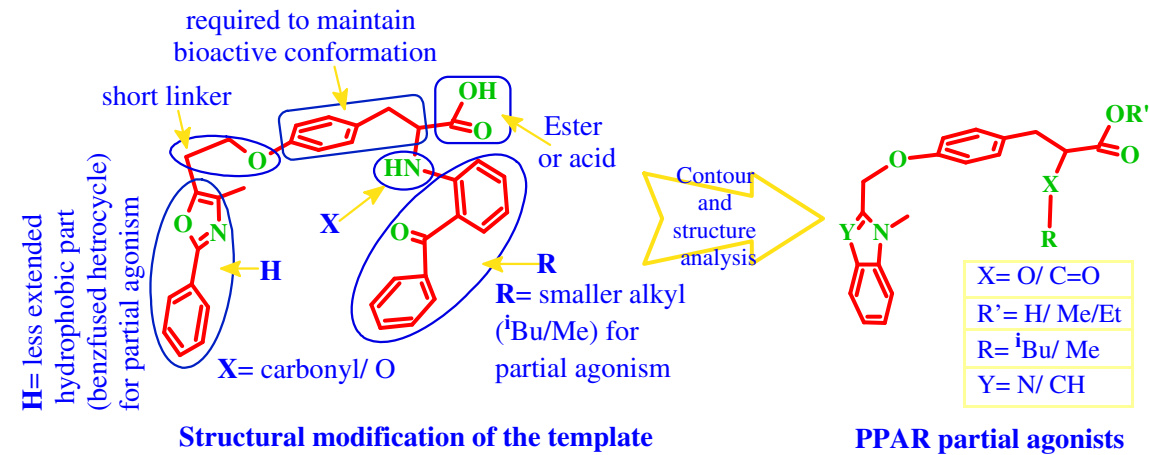

Figure 6. Pharmacophoric modifications of the template for the design of the $\alpha$ alkoxy phenyl propanoic acid and $\beta$-acetyl phenyl propanoic acid methyl/ethyl ester based NCEs (PPAR partial agonists).

part of the molecules with methyleneoxy spacer remains away from the green contours of the PPAR $\alpha$ and PPAR $\gamma$ models (figures 7 and 8). The flat aromatic ring was kept intact while design so as to maintain the bioactive ' $U$ ' conformation. We observed that a bulkier group (phenoxy, benzyloxy) at the $\alpha$-position of the acid group contributes mainly in increasing the PPAR $\gamma$ activity. Smaller alkyl groups exert less effect on the activity. Dual molecules with $\alpha$-ethoxy are known to have lesser PPAR $\gamma$ activity compared to their $\alpha$-aryloxy counterparts. The incorporation of ethoxy group into the hydrogen bonding part (as in NND9, Ragaglitazar) reduces the activity in considerable amount; we selected isobutyloxy (figures 5 and 6) to get a moderate and medium effect. This was also supported by the steric contours (green contour on the right hand side) of the PPAR $\alpha$ and PPAR $\gamma$ models (figures 3, 4, 7 and 8). The prediction of activities with the models gave expected results (table 2). The modifications consequently diminished the overall dual activity too (table 2 ). A sterically less bulky $\beta$-ketoester (methyl acetoacetate/ethyl acetoacetate) group as the hydrogen bonding part (figures 5 and 6) also gave predicted results in favour of partial agonism. Blue contours can be observed in 


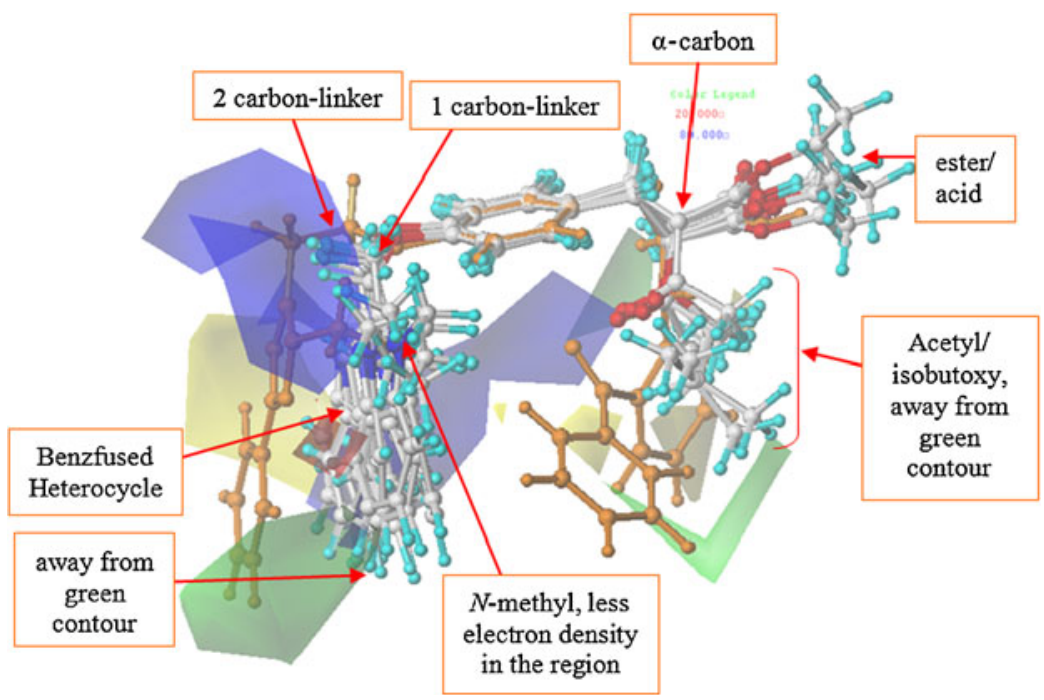

Figure 7. The reported NCEs and the template within the steric and electrostatic transparent contours of the PPAR $\alpha$ model (farglitazar shown in orange, the special modifications into the standard have been highlighted).

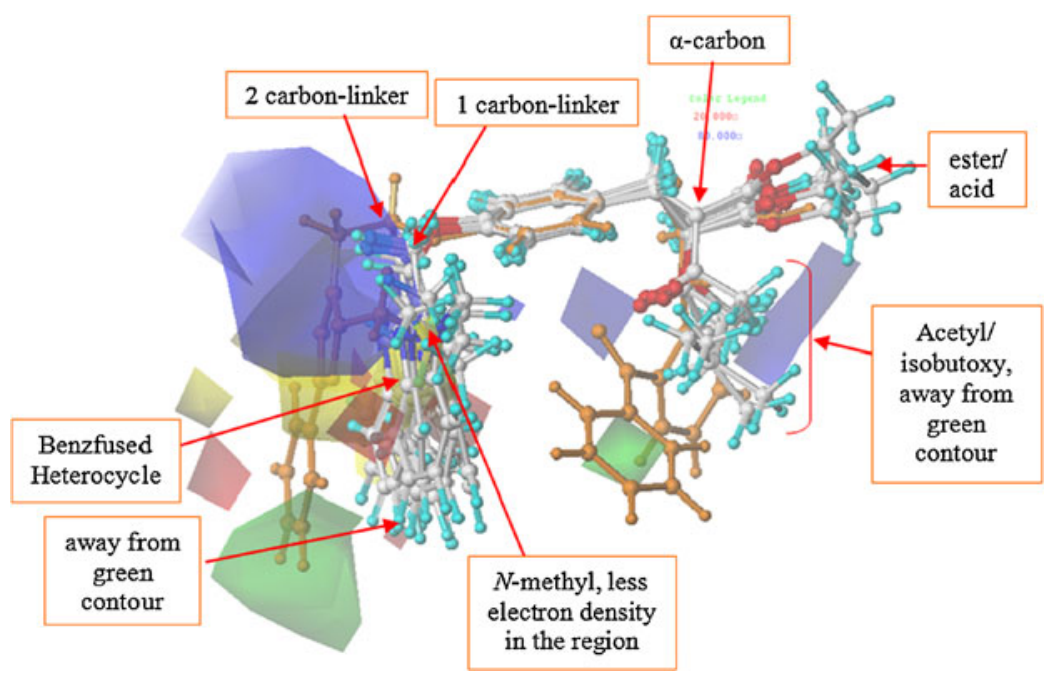

Figure 8. The reported NCEs and the template within the steric and electrostatic transparent contours of the PPAR $\gamma$ model (farglitazar shown in orange, the special modifications into the standard have been highlighted).

case of both the models near to the nitrogen atom of secondary amino group on the right hand side of the template (figures 3 and 4) which indicate positive potential in this region is favourable for enhancing $\operatorname{PPAR} \alpha$ and PPAR $\gamma$ activities, but if electron richness is provided in the region that will exert a negative effect upon the enhancement of activities. A carbonyl group in place of the amino group in the designed molecules (figures 7 and 8) served the purpose well and contributed to the reduction of activities. Though a carbonyl group can also be noticed in the template molecule at $\gamma$-postion with respect to the amino group on the right hand side but it is away from the blue contour (figures 3 and 4 ) and when the carbonyl group is kept at the $\beta$-position with respect to the ester group in the designed molecules the carbonyl oxygen atom approaches near to the blue region (figures 7 and 8 ). The antihypoglycemic activity of the 1,3-dicarbonyl compounds has been previously investigated thoroughly and reported. ${ }^{14}$ The heterocyclyl linked benzyl $\beta$-ketoester based molecules along with their benzylidene analogs were predicted for their activities (table 2) as the 


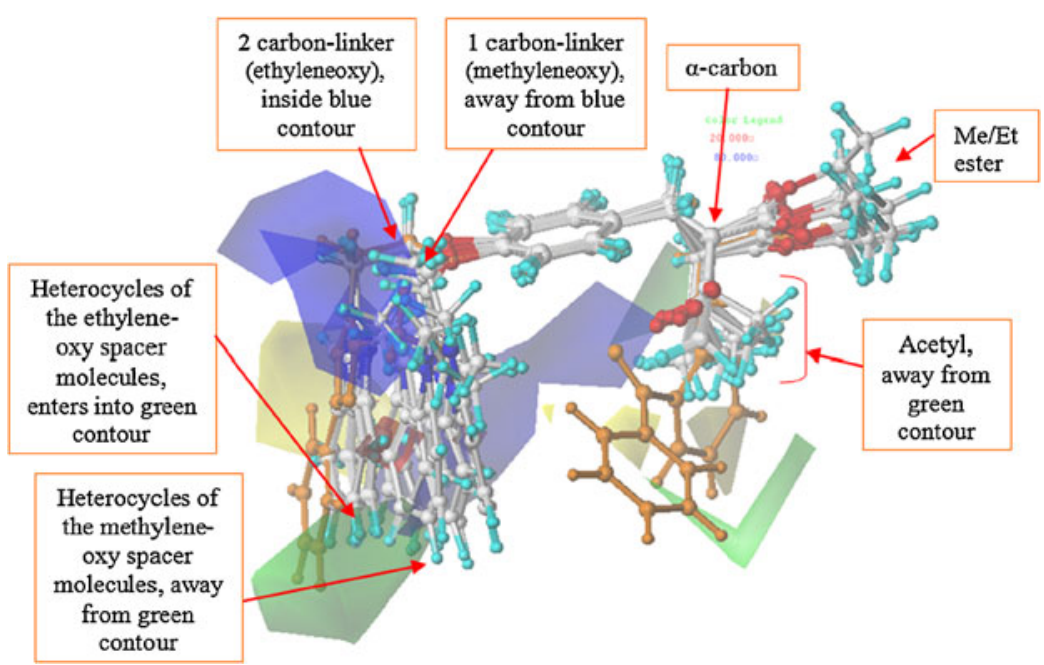

(a)

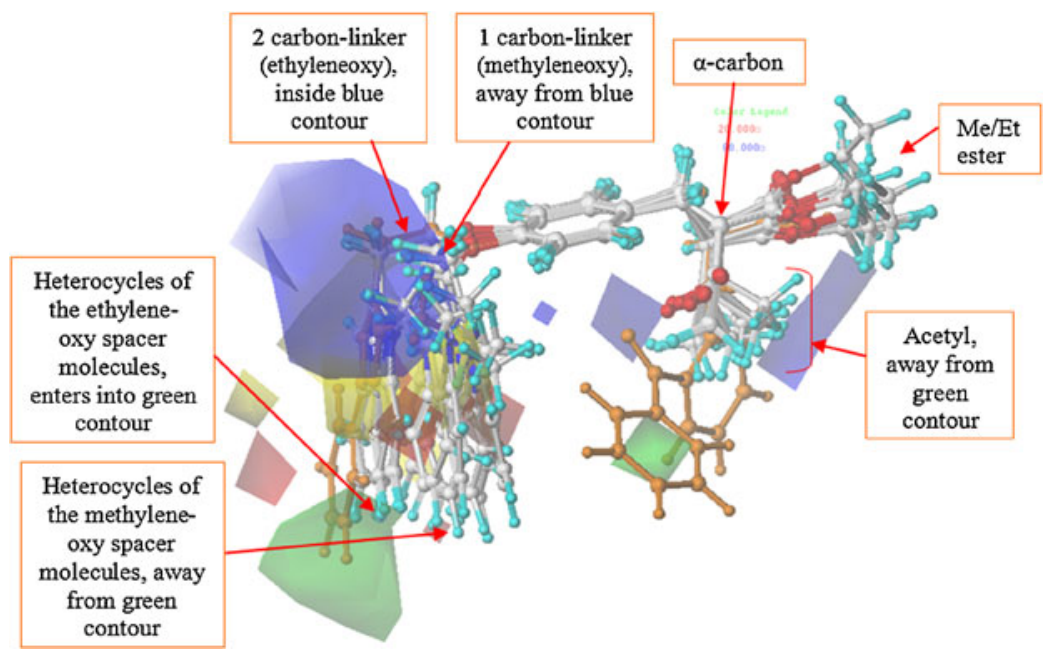

(b)

Figure 9. The reported NCEs and the template (farglitazar: orange) within the steric and electrostatic contours (transparent) of the CoMFA models,${ }^{14}$ the effects of increasing the length of the spacer are indicated. Reported $\beta$-keto ester based NCEs: (a) PPAR $\alpha$ model and (b) contours, $\operatorname{PPAR} \gamma$ model.

benzylidines are also reported to be active and show euglycemic activity. ${ }^{15}$ The benzylidene 1,3-diesters are also reported to show antihypoglycemic activity. ${ }^{14}$ The double bond can also have an effect of imparting constraint to the right hand side hydrogen bonding fragments of the designed molecules. As the diesters are reported to be active in both PPAR $\alpha$ and PPAR $\gamma$ and also exhibit lesser activities as compared to the tyrosine and $\alpha$-alkoxy dihydrocinnamic acid based agonists, ${ }^{14}$ $\beta$-ketoester was decided upon to be incorporated in the designed molecules as a part of the hydrogen bonding fragment. Electron-rich group indicating the presence of an acid was a requirement for enhancement of activity according to contour analysis. ${ }^{9}$ Ester group was introduced in a subset of designed molecules intentionally to understand the effect on reduction of activities by the modification. The designed molecules exhibited appreciable partial agonism as evident from the predicted activity data values which are lesser compared to that of the standard dual agonists like farglitazar, LY510929, GW7845 etc (table 2). The molecules with methyleneoxy spacer were found to be of lesser predicted activities compared to the molecules with ethyleneoxy spacer and hence preferred over the latter for selection for synthesis. The lesser predicted activities of the molecules with methyleneoxy spacer may be attributed to the shortened length of the spacer which in turn reduces the distance between the hydrophobic 


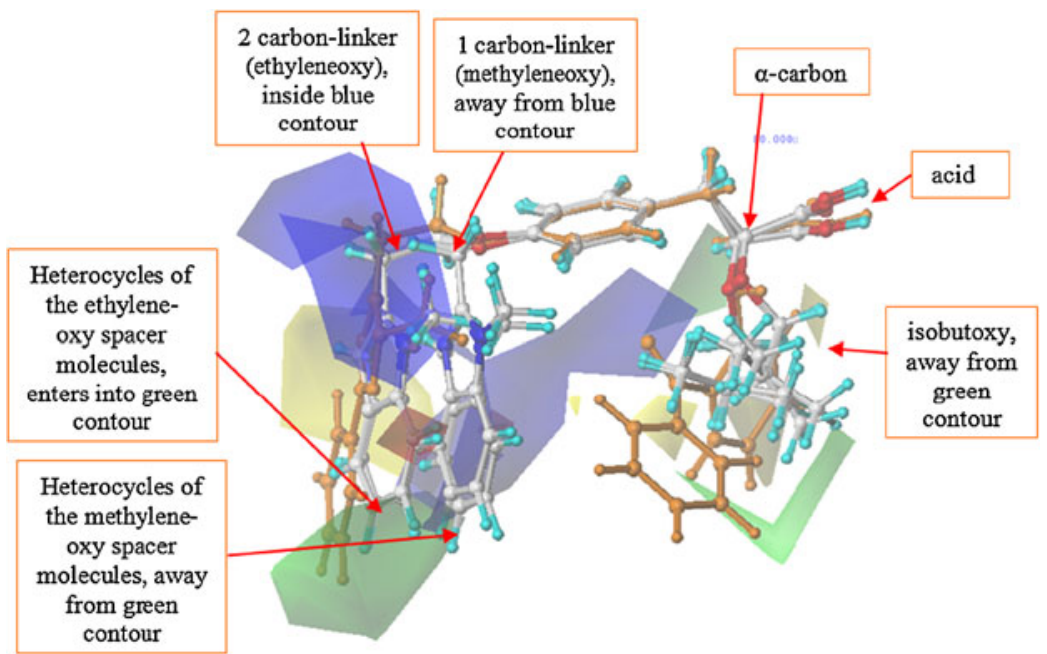

(a)

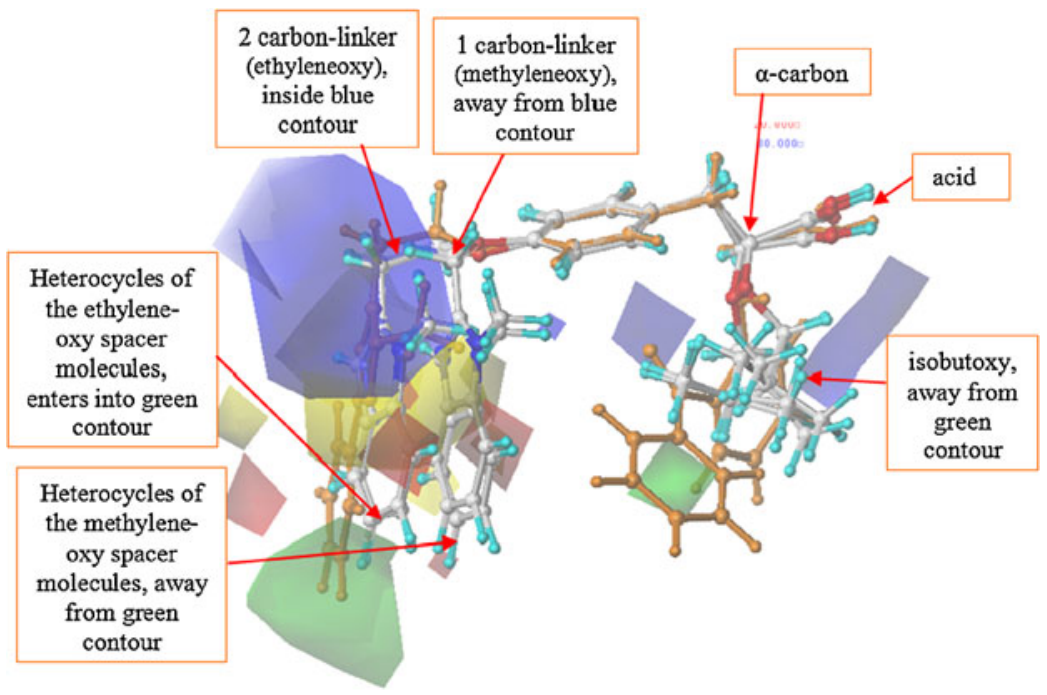

(b)

Figure 10. The reported NCEs and the template (farglitazar: orange) within the steric and electrostatic contours (transparent) of the CoMFA models, ${ }^{14}$ the effects of increasing the length of the spacer are indicated. Reported $\alpha$-alkoxy acid based NCEs: (a) contours, PPAR $\alpha$ model and (b) contours, PPAR $\gamma$ model.

part and the hydrogen bonding tail and introduces a constraint to the molecules that helps lesser binding interactions at the receptor site compared to the molecules with ethyleneoxy spacer as well as the standard molecules. Smaller hydrophobic and hydrogen bonding units with moderate bulk were introduced to impart features of partial agonism to the designed NCEs. The feature of partial agonism of the NCEs was further validated computationally by the docking studies at PPAR $\gamma \mathrm{X}$-ray crystal structure. This showed less binding affinities of the designed NCEs to the PPAR $\gamma$ active site as compared to the previously reported dual agonists as evident from the lesser docking score and Gold score ${ }^{16,17}$ values (table 2) and they exhibited lesser number of hydrogen bond interactions as compared to the previously discussed dual agonists (table 3). From the predicted activities and binding affinities it is evident that the molecules with methyleneoxy linker exhibit lesser activities and binding affinities compared to their ethyleneoxy counterparts in almost all the cases so the molecules with methyleneoxy linker were selected as better partial agonists for synthesis. The syntheses and characterization of the $\alpha$-isobutoxy propanoic acid based and the $\beta$-ketoester based compounds are reported in the present work.

The ketoester final NCEs were achieved by first synthesizing the left hand side (lhs) alcohol units 
(schemes 1 and 2) and then right hand side (rhs) phenolic units (scheme 3 ) followed by coupling the two units by Mitsunobu reaction (scheme 3). The final $\alpha$-alkoxy acids were achieved from the aldehydes $(\mathbf{1 a} / \mathbf{2 b})$ obtained from the lhs alcohols $(\mathbf{1 / 2 a})$ by a sequence of four steps of synthesis (scheme 4).

The benzimidazolyl linked aldehyde (1a) was prepared by the synthetic route as shown in scheme 1 . $N$-methyl-1, 2-phenylene diamine dihydrochloride was cyclised drastically with glycolic acid to furnish the corresponding hydroxyl methyl compound (1) in excellent yield. This was reacted with 4-fluorobenzaldehyde to afford the aldehyde (1a). The hydroxyl methyl compound (2a) was prepared by a sequence from commercially available ethyl indole-2-carboxylate via $\mathrm{N}$-methylation and subsequent reduction (scheme 2). The yields of the N-methylated product (2a) and the reduced product (2a) were very good. The indolyl linked aldehyde (2b) was prepared from (2a) in a similar way as shown in scheme1 for (1a). The prepared compounds (1-2b) were characterized and confirmed by IR, ${ }^{1} \mathrm{HNMR}$ and mass spectroscopy.

The ketoesters rhs units $(\mathbf{4 a} / \mathbf{b}$ and $\mathbf{6 a} / \mathbf{b})$ were synthesized as shown in scheme 3. Commercially available p-anisaldehyde and methyl acetoacetate/ethyl acetoacetate were condensed by Knoevenagel rection in the presence of a base to yield the methoxy benzylidene compounds $\mathbf{3 a} / \mathbf{b}$ which were reduced catalytically in a Parr hydrogenator to get the methoxy benzyl compounds $\mathbf{5 a} / \mathbf{b}$. Demethylation of $\mathbf{3 a} / \mathbf{b}$ and $\mathbf{5 a} / \mathbf{b}$ with boron tribromide gave the corresponding phenolic benzylidene-based and phenolic benzyl-based compounds $(\mathbf{4 a} / \mathbf{b}$ and $\mathbf{6 a} / \mathbf{b}$, respectively) which are the rhs units for the synthesis of the $\beta$-ketoester based final NCEs. The methoxy benzylidenes $\mathbf{3 a} / \mathbf{b}$ and hydroxyl benzylidenes $\mathbf{4 a} / \mathbf{b}$ were obtained as mixture of geometric isomers which was clearly indicated by their PMR spectra. $4 \mathbf{a} / \mathbf{b}$ and $\mathbf{6 a} / \mathbf{b}$ were coupled with the lhs units 1a and 2a by Mitsunobu process to finally synthesize the $\beta$-ketoester based final NCEs (7a-10b, scheme 3 ). The benzylidene $\beta$-ketoester-based final NCEs (7a-8b) were also obtained as mixture of geometrical isomers as evident from their PMR spectra and was as expected.

The efficient syntheses of the $\alpha$-alkoxy acids have been shown in scheme 4 . The benzimdazolyl linked aldehyde (1a) and the indolyl linked benzaldehyde (2b) were treated (separately) with methoxymethyl triphenylphosphonim chloride in the presence of a strong base lithium diisopropyl amine (prepared in situ by mixing butyl lithium and isopropyl amine at low temperature) to get $\mathbf{1 1 a} / \mathbf{b}$ in good yields. The diacetals $\mathbf{1 2 a} / \mathbf{b}$ were synthesized from $\mathbf{1 1 a} / \mathbf{b}$ by refluxing with isobutanol catalysed by $p$-toluenesuphonic acid.
The isobutoxy cyanides $(\mathbf{1 3 a} / \mathbf{b})$ were obtained in appreciable yields by reacting $\mathbf{1 2 a} / \mathbf{b}$ with trimethylsilyl cyanide. Finally, the $\alpha$-alkoxy acid based final NCEs $(\mathbf{1 4 a} / \mathbf{b})$ were achieved in excellent yields by hydrolysing the cyanides $(\mathbf{1 3 a} / \mathbf{b})$.

\section{Conclusion}

We report here the syntheses and characterization, benzimidazole and indole linked benzyl based $\alpha$-alkoxy propanoic acids and benzylidene and benzyl based $\beta$ ketoesters as partial PPAR $\alpha / \gamma$ agonists as anti type 2 diabetic compounds. The activities and binding affinities at individual PPAR of synthesized molecules were predicted to support the design of the molecules with expected partial agonism.

\section{Acknowledgements}

The authors are thankful to Head, Department of Chemistry, Punjabi University and are also grateful to the Director, Regional Sophisticated Instrumentation Centre (RSIC), Panjab University, Chandigarh and Director, National Institute of Pharmaceutical Education and Research (NIPER), SAS Nagar, Mohali, Punjab for extending the facilities for the analysis of various compounds reported in this paper. We greatly acknowledge the financial support from IndSwift Labs. Ltd., SAS Nagar, Mohali, Punjab for the ISLL-Punjabi University Collaborative Research Project, and providing the research fellowship to two of the authors VK and PG and for the timely guidance and support.

\section{References}

1. Ahmed I, Furlong K, Flood J, Treat P V and Goldstein J B 2007 Am. J. Ther. 1449

2. Balakumar P, Madhankumar R, Subrahmanya S G, Krishan P and Singh M 2007 Pharmacol. Res. 5691

3. Fiévet C, Fruchart J C and Staels B 2006 Curr. Opin. Pharmacol. 6606

4. Nissen S E, Wolski K and Topol E J 2005 JAMA 294 2581

5. Hellmold H, Zhang H, Andersson U, Blomgren B, Holland T, Berg A, Elebring M, Sjögren N, Bamberg K, Dahl B, Westerberg R, Dillner B, Tugwood J, Roberts R, Lundholm E, Camejo G, Skånberg I and Evans J 2007 Toxicol. Sci. 9863

6. Haberman A B, Haberman Associates. The Biopharmaceutical Consortium, Sep 20, 2006

7. Burgermeister E, Schnoebelen A, Flament A, Benz J, Stihle M, Gsell B, Rufer A, Ruf A, Kuhn B, Märki H P, Mizrahi J, Sebokova E, Niesor E and Meyer M 2006 Mol. Endocrinol. 20809 
8. Fujimura T, Kimura C, Oe T, Takata Y, Sakuma H, Aramori I and Mutoh S 2006 J. Pharmacol. Exp. Ther. 318863

9. Verma R, Ghosh P, Kumar V and Wadhwa L K 2011 Med. Chem. Res. doi: 10.1007/s00044-011-9818-7

10. Cai Z, Feng J, Guo Y, Li P, Shen Z, Chu F and Guo Z 2006 Bioorg. Med. Chem. 14866

11. Kim N, Lee K, Koo B, Li F, Yoo J, Park H, Min K, Lim J, Kim M, Kim J and Suh Y 2007 Bioorg. Med. Chem. Lett. 173595

12. Kuhn B, Hilpert H, Benz J, Binggeli A, Grether U, Humm R, Märki H, Meyer M and Mohr P 2006 Bioorg. Med. Chem. Lett. 144016
13. Cronet P, Petersen J, Folmer R, Blomberg N, Sjöblom K, Karlsson U, Lindstedt E and Bamberg K 2001 Structure 9699

14. Shinkai H, Onogi S, Tanaka M, Shibata T, Iwao M, Wakitani K and Uchida I 1998 J. Med. Chem. 411927

15. Lohray B B, Bhushan B, Rao B P, Madhavan G R, Murali N, Rao K N, Reddy A K and Rajesh B M 1998 J. Med. Chem. 411619

16. Sybyl 7.3 (2006), 1699 Hanley Road, St. Louis, MO 63144, USA

17. Suh Y, Kim N, Koo B, Lee K, Moon S, Shin D, Jung J, Paek S, Chang D, Li F, Hyun K, Ryu J and Hyun P 2008 J. Med. Chem. 516318 